\title{
Brioflora do Parque Estadual Intervales, São Paulo, Brasil: uma importante área para conservação da biodiversidade da Mata Atlântica do Sudeste brasileiro Bryoflora from the Intervales State Park, São Paulo, Brazil: an important area for the biodiversity conservation of the Brazilian Southeastern Atlantic forest
}

\author{
Sandra Regina Visnadi \\ Instituto de Botânica. São Paulo, São Paulo, Brasil
}

\begin{abstract}
Resumo: O Parque Estadual Intervales (PEI) forma, com outras unidades de conservação, o Continuum Ecológico de Paranapiacaba, que é uma das sete maiores áreas contínuas de Mata Atlântica do país. $\bigcirc$ trabalho tem por objetivo evidenciar a riqueza da brioflora, pois lista 199 espécies (90\%), além das 21 já citadas na literatura para o PEl, totalizando 220 espécies para a Mata Atlântica de encosta (floresta ombrófila densa) desse parque. $\bigcirc$ material totaliza 767 exsicatas, que se encontram depositadas no Herbário Maria Eneyda Pacheco Kauffmann Fidalgo, do Instituto de Botânica de São Paulo (SP). A brioflora do PEl é rica e característica da Mata Atlântica de encosta do estado de São Paulo, mas com algumas espécies restritas ao parque, não ocorrendo nesse ecossistema de outras localidades paulistas; inclui duas citações novas para o Brasil (Chryso-hypnum squarrosulum, Leskeodon andicola) e cinco para São Paulo (Fissidens dendrophilus, Macromitrium longifolium, Orthostichopsis praetermissa, Pelekium scabrosulum e Thamniopsis cruegeriana), além de possuir 13 espécies endêmicas do Brasil e uma espécie que, no Brasil, restringe-se ao estado paulista. Esses dados demonstram a importância do PEI para a conservação e proteção da diversidade da brioflora local, do estado de São Paulo e do Brasil.
\end{abstract}

Palavras-chave: Antóceros. Hepáticas. Musgos. Mata Atlântica de encosta.

Abstract: The Intervales State Park, together with other protected areas form the Ecological Continuum of Paranapiacaba, which is one of the seven largest continuous areas of the Brazilian Atlantic forest. The paper aims to show the richness of the bryoflora, because it list 199 species (90\%) in addition of the 21 species already recorded in literature for PEI, totalizing 220 species for the hillside Atlantic forest (ombrophilous dense forest) of this park. The material totalizes 767 exsiccate, which are deposited in the Maria Eneyda Pacheco Kauffmann Fidalgo Herbarium, of the São Paulo Botanical Institute (SP). The bryoflora from PEI is rich and characteristic of the hillside Atlantic Forest of the São Paulo state, but with some species restricted to the park, not occurring in this ecosystem of other São Paulo localities; it includes two new records for Brazil (Chryso-hypnum squarrosulum, Leskeodon andicola) and five for São Paulo (Fissidens dendrophilus, Macromitrium longifolium, Orthostichopsis praetermissa, Pelekium scabrosulum, and Thamniopsis cruegeriana), besides having 13 endemic species from Brazil and one species, which in Brazil is restricted to the São Paulo state. These data demonstrate the importance of the PEI in the conservation and protection of the bryophytes diversity of the site, São Paulo state and Brazil.

Keywords: Hornworts. Liverworts. Mosses. Hillside Atlantic forest.

VISNADI, S. R., 2015. Brioflora do Parque Estadual Intervales, São Paulo, Brasil: uma importante área para conservação da biodiversidade da Mata Atlântica do Sudeste brasileiro. Boletim do Museu Paraense Emílio Goeldi. Ciências Naturais 10(1): 105-125.

Autor para correspondência: Sandra Regina Visnadi. Instituto de Botânica. Centro de Pesquisa em Plantas Avasculares e Fungos. Núcleo de Pesquisa em Briologia. Av. Miguel Stéfano, 3687 - Água Funda. São Paulo, SP, Brasil. CEP $04301-012$ (svisnadi@uol.com.br).

Recebido em 22/10/2014

Aprovado em 11/05/2015

Responsabilidade editorial: Anna Luiza Ilkiu-Borges

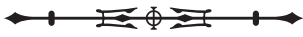




\section{INTRODUÇÃO}

O Parque Estadual Intervales (PEI) compõe parcela importante da reserva da biosfera da Mata Atlântica, pois forma, com outras unidades de conservação, o Continuum Ecológico de Paranapiacaba, uma das sete maiores áreas contínuas de Mata Atlântica identificadas no país, com mais de 120 mil hectares de extensão (Sallun Filho et al., 2010; Ivanauskas et al., 2012; Sarfati \& Sano, 2012).

O PEl localiza-se na serra de Paranapiacaba, entre os vales dos rios Paranapanema e Ribeira de Iguape, abrangendo parte dos municípios de Eldorado Paulista, Guapiara, Iporanga, Ribeirão Grande e Sete Barras, além de Apiaí e Capão Bonito, na zona de Amortecimento (24 12' a $24^{\circ} 32^{\prime} \mathrm{S}$ e $48^{\circ} 03^{\prime}$ a $48^{\circ} 32^{\prime}$ ' W), ao sul do estado de São Paulo, onde ocupa uma área de 41.704, 27 hectares (Pepinelli et al., 2005; Sallun Filho et al., 2010; Ivanauskas et al., 2012; Sarfati \& Sano, 2012).

O parque é uma Unidade de Proteção Integral da natureza paulista, pois preserva seus ecossistemas naturais, que possuem grande relevância ecológica e beleza cênica (SNUC, 2004; Rodrigues et al., 2008) e encontra-se sob administração da Fundação para a Conservação e a Produção Florestal do Estado de São Paulo - Fundação Florestal, vinculada à Secretaria Estadual do Meio Ambiente.

As terras da Fazenda Intervales tornaram-se parque estadual, com área delimitada, através dos decretos estaduais de $n^{\circ}$ 40.135 de 08/06/1995 e de n 44.293 de 04/10/1999, e da lei estadual de no 10.850, de 06/07/2001, que consideram prioritários o desenvolvimento sustentável regional e a conservação da natureza no Vale do Ribeira, com extensas áreas de mananciais, significativos sítios espeleológicos e frágeis encostas cobertas pela Mata Atlântica (Rodrigues et al., 2008; Sallun Filho et al., 2010; Sarfati \& Sano, 2012).

O PEI apresenta clima subtropical de altitude, sem estação seca, com alta precipitação (2.000 a 3.000 mm/ano) e temperatura média variando entre 17 e $19^{\circ} \mathrm{C}$; é coberto, principalmente, por Mata Atlântica de encosta, com pouca intervenção antrópica e enquadra-se na região ecológica da floresta ombrófila densa (Pepinelli et al., 2005; Zipparro et al., 2005).
- parque localiza-se em áreas com rochas carbonáticas, nas quais o relevo cárstico se caracteriza pela ocorrência expressiva de cavernas. Os impactos causados pela mineração, que se instalou na região há anos, referem-se à supressão da vegetação, alteração do relevo, dos cursos d'água e do regime hidrológico subterrâneo, à destruição de cavidades naturais e ao deslocamento de fauna. Não ocorrem concentrações urbanas significativas na área, mas a própria ocupação e a agricultura são potencialmente poluidoras. $\bigcirc$ PEI tem conseguido conciliar o ecoturismo com a preservação ambiental, pois também apresenta 13 cachoeiras, dois mirantes e pode ser percorrido através de 25 roteiros de visitação, mas apenas parte da área total do parque é aberta à visitação pública. Além disso, a infraestrutura turística do PEI é limitada, o que naturalmente restringe o número de visitantes (Sallun Filho et al., 2010; Sarfati \& Sano, 2012).

A região atlântica costeira é a mais densamente povoada do Brasil, onde grande parte da vegetação já desapareceu e as florestas que ainda persistem encontram-se ameaçadas (Gradstein \& Costa, 2003). Apenas 9,3 dos 11,73\% da vegetação remanescente da Mata Atlântica estão protegidos em unidades de proteção integral. Adicionado a isso, somente no sudeste de São Paulo e nordeste do Paraná ocorre a floresta ombrófila densa sobre regiões cársticas. Nessas áreas, em que o PEI se localiza, as lentes de calcário apresentam geomorfologia diferenciada, os solos são mais férteis, com altos teores de cálcio e magnésio, e as florestas se distinguem do padrão encontrado sobre os solos mais ácidos, menos férteis e com teores de alumínio mais elevados, característicos das encostas da Serra do Mar (Ivanauskas et al., 2012). Todavia, apenas 11 famílias com 21 espécies de briófitas foram registradas, até o momento, para a Mata Atlântica do PEI (Buck \& Vital, 1992; Crum, 1992; Schäfer-Verwimp, 1992; Visnadi, 2002; Gradstein \& Costa, 2003; Yano, 2010, 2011, 2013). Portanto, o presente artigo tem por objetivo evidenciar a riqueza da brioflora desse parque, ampliando a lista de espécies de briófitas para essa localidade com Mata Atlântica, no estado de São Paulo.

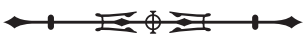




\section{MATERIAL E MÉTODOS}

O trabalho foi realizado no Parque Estadual Intervales (PEI), estado de São Paulo, Brasil. O ecossistema estudado refere-se à Mata Atlântica de encosta, que se enquadra na região ecológica da floresta ombrófila densa, como relacionado anteriormente.

O estudo da brioflora incluiu as 21 espécies registradas pela literatura já citada e o material do acervo do Herbário Maria Eneyda Pacheco Kauffmann Fidalgo, do Instituto de Botânica de São Paulo (SP). Esse material foi coletado por A. Schäfer-Verwimp, D. M. Vital, I. Verwimp, S. R. Visnadi e W. R. Buck, em solo, rocha, estalactite, na vegetação (casca de forófitos vivos ou mortos e folhas), em ninho abandonado de pássaro, caído no solo e em construções (parede de alvenaria, telhado), ao longo de trilhas ou estradas estreitas, abertas dentro da mata, totalizando 767 exsicatas, cujo voucher para cada espécie foi relacionado no Apêndice 1.

A identificação do material foi baseada na bibliografia especializada e nos materiais depositados no Herbário SP. Materiais de Orthostichella pentasticha (Brid.) W. R. Buck, já relacionados para o PEI (Visnadi, 2002), foram reidentificados, parte como O. pachygastrella (Müll. Hal. ex Ångstr.) B.H. Allen \& Magill e parte como O. versicolor (Müll. Hal.) B. H. Allen \& W. R. Buck, segundo Allen \& Magill (2003, 2007).

As espécies foram listadas por ordem alfabética em divisões, famílias, gêneros, espécies, subespécies e variedades, segundo classificação de Renzaglia et al. (2009), para Anthocerotophyta, Goffinet et al. (2009), para Bryophyta, e Crandall-Stotler et al. (2009), para Marchantiophyta (Apêndice 1).

A citação do nome das espécies e as informações referentes à distribuição geográfica e à ocorrência na Mata Atlântica foram baseadas no banco de dados de briófitas do Instituto de Pesquisas Jardim Botânico do Rio de Janeiro (Costa \& Peralta, 2014), do Jardim Botânico de Missouri (Tropicos, s. d.) e do Conservatório e Jardim Botânico de Genebra (Index Hepaticarum, s. d.), além da literatura já mencionada, acrescida de Hell (1969), Sim (1973), Crum \&
Anderson (1981), Nishimura \& Ando (1986), Frahm (1991), Schäfer-Verwimp (1991), Ireland (1992), Reese (1993), Yamaguchi (1993), Allen (1994, 2002, 2010), Sharp et al. (1994), Florschütz-de Waard (1996), Visnadi \& Vital (1997, 2000), Buck (1998), Magill \& van Rooy (1998), Oliveira-e-Silva \& Yano (2000), Gradstein et al. (2001), Kruijer (2002), Meagher \& Fuhrer (2003), Wigginton (2004), Visnadi (2005, 2006, 2011, 2012, 2013a, 2013b), Flora of North America Editorial Committee (2007), Porley (2008), Santos \& Costa (2008), Yano (2008), Alvarenga et al. (2010), Silva \& Pôrto (2010), Costa et al. (2011), Machado \& Luizi-Ponzo (2011), Peralta \& Yano (2011, 2012), Santos et al. (2011), Bordin \& Yano (2013), Glime (2013) e Valente et al. (2013a, 2013b).

Os dados referentes ao grupo ecológico e às formas de vida das briófitas foram tomados durante a realização do trabalho de campo e do estudo do material coletado, da literatura e dos bancos de dados, já relacionados anteriormente. O status de conservação das oito espécies com distribuição restrita no Brasil foi avaliado segundo diretrizes da IUCN (s. d.).

Finalmente, analisou-se o grau de similaridade da brioflora da Mata Atlântica do PEI com a brioflora da Mata Atlântica (floresta ombrófila densa) de Ubatuba, Caraguatatuba, São Sebastião, Bertioga, Mogi das Cruzes, Santo André e Iguape, tomando-se parte das informações disponíveis em Visnadi (2005, 2012), Peralta \& Yano (2008, 2012), Yano et al. (2009), Santos et al. (2011) e Yano (2013) sobre essas sete últimas localidades paulistas. Posteriormente, aplicou-se o índice de Sørensen à matriz de dados binários (espécies $\times$ sete localidades, já citadas, e PEI), a fim de se realizar a análise do agrupamento, por meio o método de ligação pela média de grupo, utilizando-se o Programa Fitopac 2.1 (Shepherd, 2009); esses procedimentos foram também realizados para a matriz de dados binários sem as espécies restritas ao parque, considerando as mesmas sete localidades e o PEI, a fim de verificar se a exclusão dessas espécies altera o padrão de variação dos dados entre os locais considerados. 


\section{RESULTADOS E DISCUSSÃO}

Para o PEI, foram listadas 54 famílias, com 126 gêneros e 220 espécies, três subespécies e sete variedades de briófitas (Apêndice 1). A brioflora do Parque é rica, pois esses dados representam $61 \%$ das 88 famílias, $45 \%$ dos 278 gêneros e 25\% das 889 espécies registradas para o estado de São Paulo (Costa \& Peralta, 2014).

A lista inclui 199 espécies, além das 21 já citadas na literatura para a brioflora da Mata Atlântica do PEI, sendo que esse acréscimo corresponde a $90 \%$ das 220 espécies ora listadas para a área de estudo. A brioflora pode ser efetivamente protegida apenas pela preservação de seu habitat (Gradstein et al., 2001). Portanto, as 220 espécies listadas para o PEI evidenciam a relevância do parque na conservação e na proteção da flora de briófitas da Mata Atlântica local.

Anthocerotophyta totaliza duas famílias, dois gêneros, duas espécies e uma subespécie, em apenas 1\% das amostras. Em muitas regiões do planeta, o número de táxons de musgos é maior que o registrado para hepáticas e antóceros (Tan \& Pócs, 2000), como no PEI, no qual Bryophyta é composta por 34 famílias, 81 gêneros, 133 espécies, uma subespécie e sete variedades, tendo sido registrada no maior número de amostras (60\%). Em outras unidades de conservação paulistas, também situadas mais no interior do estado, ao longo de serras e com Mata Atlântica, como o Parque Natural Municipal Francisco Affonso de Mello (Peralta \& Yano, 2012) e o Parque Estadual Turístico do Alto do Ribeira (PETAR) (Visnadi, 2013b), musgos superam hepáticas em número de espécies, exceto na Reserva Biológica do Alto da Serra de Paranapiacaba (Yano et al., 2009), onde se observa o contrário. Marchantiophyta está representada por menos táxons (18 famílias, 43 gêneros, 85 espécies e uma subespécie), em menor percentagem de amostras (39\%). Todavia, a família que se destaca pelos maiores números de gêneros (20) e de espécies (36), no PEI, é Lejeuneaceae, já registrada na literatura como a maior família de hepáticas (Crandall-Stotler et al., 2009; Martinelli \& Moraes, 2013), principalmente tropical (Richards, 1984), e que predomina nos manguezais, nas restingas, áreas antrópicas, na Mata Atlântica de encosta e em picos paulistas (Visnadi, 2005, 2008, 2009, 2010, 2011, 2012, 2013a, 2013b; Yano et al., 2009; Santos et al., 2011).

Em florestas tropicais úmidas, a maioria das briófitas é epífita e, especificamente, corticícola (Richards, 1984; Gradstein et al., 2001; Santos \& Costa, 2008), como no PEI, em que o substrato mais disponível para 202 espécies é casca de forófitos vivos (63\% das espécies em $45 \%$ das amostras) (Figura 1, Apêndice 1).

Briófitas terrestres são mais comuns em florestas de encosta do que em florestas de planície (Gradstein et al., 2001). Essas plantas são também mais frequentes no PEI (38\% das espécies em 21\% das amostras, Figura 1, Apêndice 1) e no PETAR (23\% das espécies em 11\% das amostras, Visnadi, 2012), onde a floresta ombrófila densa cobre o relevo montanhoso da serra de Paranapiacaba, e bem mais raras para as formações florestais não inundáveis das restingas do estado de São Paulo (3\% das espécies, Visnadi, 2010).

PETAR e PEI são dois parques circunvizinhos e situados sobre regiões cársticas (Ivanauskas et al., 2012). Todavia, rocha é o substrato mais disponível para a brioflora do PETAR (54\% das espécies em 31,5\% das amostras, segundo Visnadi, 2012), não para a brioflora do PEI (20\% das espécies em $8 \%$ das amostras, Figura 1, Apêndice 1), que predomina em casca de forófitos vivos, como relacionado anteriormente. O PETAR é coberto por Mata Atlântica, com trechos de vegetação secundária em regeneração e outros alterados pela presença de bambus; também apresenta campo antrópico, afloramentos de rocha e áreas de uso antrópico (Ivanauskas et al., 2012; Visnadi, 2013b). O PEI é mais conservado em relação ao PETAR, pois nele a visitação é mais controlada e limitada, apresentando Mata Atlântica de encosta com pouca intervenção antrópica, e tem conseguido conciliar o ecoturismo com a preservação ambiental (Zipparro et al., 2005; Sarfati \& Sano, 2012). 


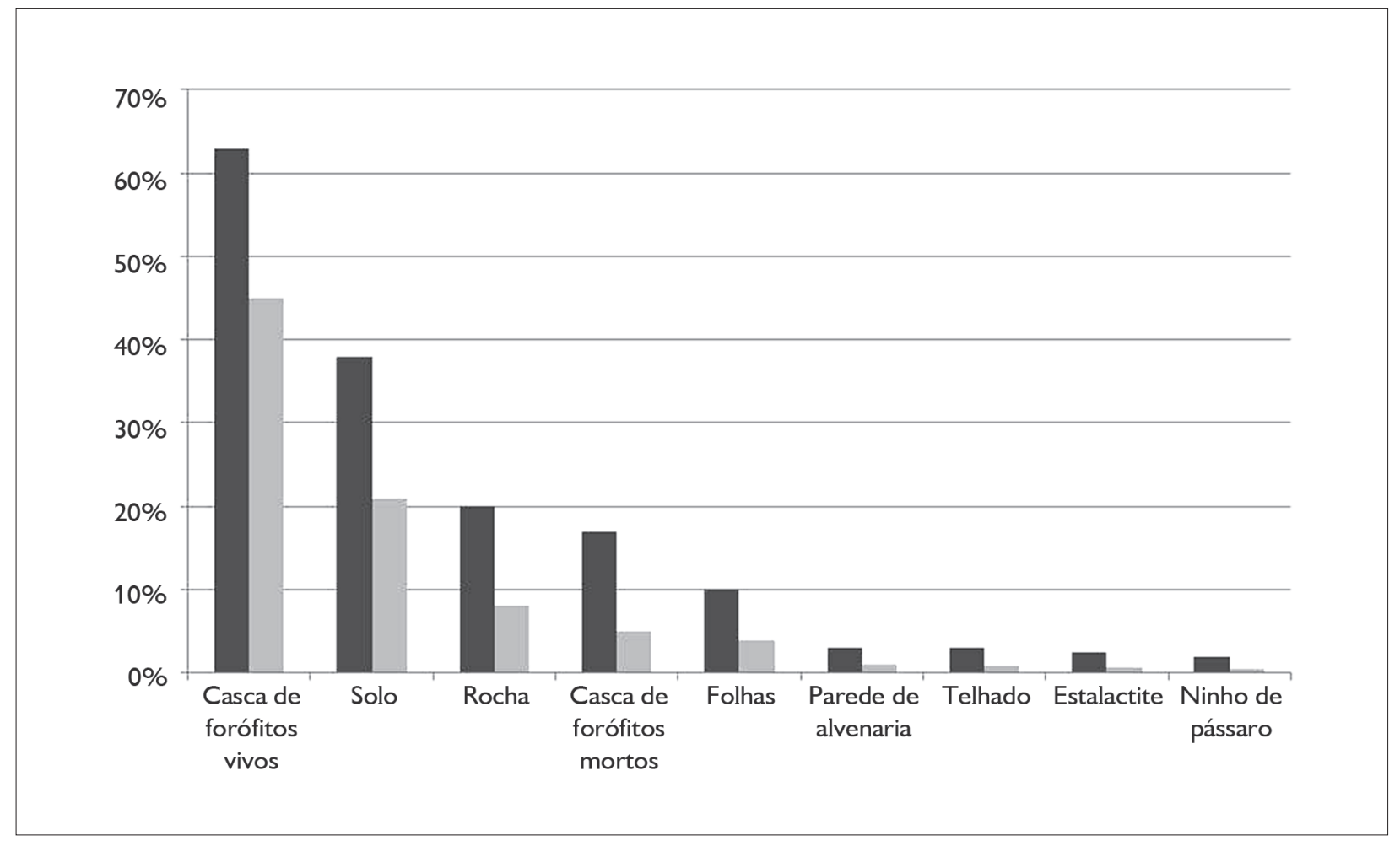

Figura 1. Percentagem de espécies (colunas escuras) e de amostras (colunas claras) de briófitas para cada tipo de substrato encontrado no Parque Estadual Intervales (PEI), São Paulo.

As espécies foram menos frequentemente encontradas em casca de forófitos mortos ( $17 \%$ das espécies em $5 \%$ das amostras) e, raramente, em folhas (10\% das espécies em 4\% das amostras), parede de alvenaria (3\% das espécies em $1 \%$ das amostras), telhado (3\% das espécies em 0,9\% das amostras), estalactite (2,5\% das espécies em 0,6\% das amostras) e ninho de pássaro (2\% das espécies em 0,5\% das amostras) (Figura 1, Apêndice 1).

Lejeuneaceae folhosas são as epíflas mais frequentes (Bates, 2009), sendo que algumas delas são exclusivas desse tipo de substrato (Gradstein et al., 2001), inclusive no PEI, onde há um total de dez das 20 espécies registradas para folhas vivas (Cololejeunea cardiocarpa, C. obliqua, Diplasiolejeunea cavifolia, D. rudolphiana, Drepanolejeunea bidens, D. biocellata, Lejeunea glaucescens, L. raddiana, Microlejeunea bullata e Odontolejeunea lunulata). Outras três espécies exclusivamente epíflas no local são Crossomitrium patrisiae, Syrrhopodon parasiticus (musgos) e Metzgeria aurantiaca (hepática). Apenas sete espécies, Floribundaria flaccida, Lepidopilidium aureo-purpureum, Meteoridium remotifolium, Zelometeorium patulum (musgos), Drepanolejeunea mosenii, Plagiochila gymnocalycina e P. martiana (hepáticas), foram também registradas para solo, rocha, casca de forófitos vivos ou mortos, ninho de pássaro e parede de alvenaria (Apêndice 1).

Em São Paulo, espécies restritas a um tipo de substrato predominam em manguezais, restingas, picos, áreas antrópicas, na Mata Atlântica de encosta (Visnadi, 2005, 2008, 2009, 2010, 2011, 2012, 2013a, 2013b) e no PEI, onde representam 55\% das espécies. As briófitas foram menos frequentemente encontradas em dois (25\% das espécies) e, raramente, em três (7,8\% das espécies), quatro (3,7\% das espécies) e cinco $(0,5 \%$ das espécies, Orthostichella pachygastrella) substratos distintos (Figura 2). Para Peralta \& Yano (2008), a brioflora

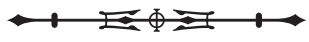




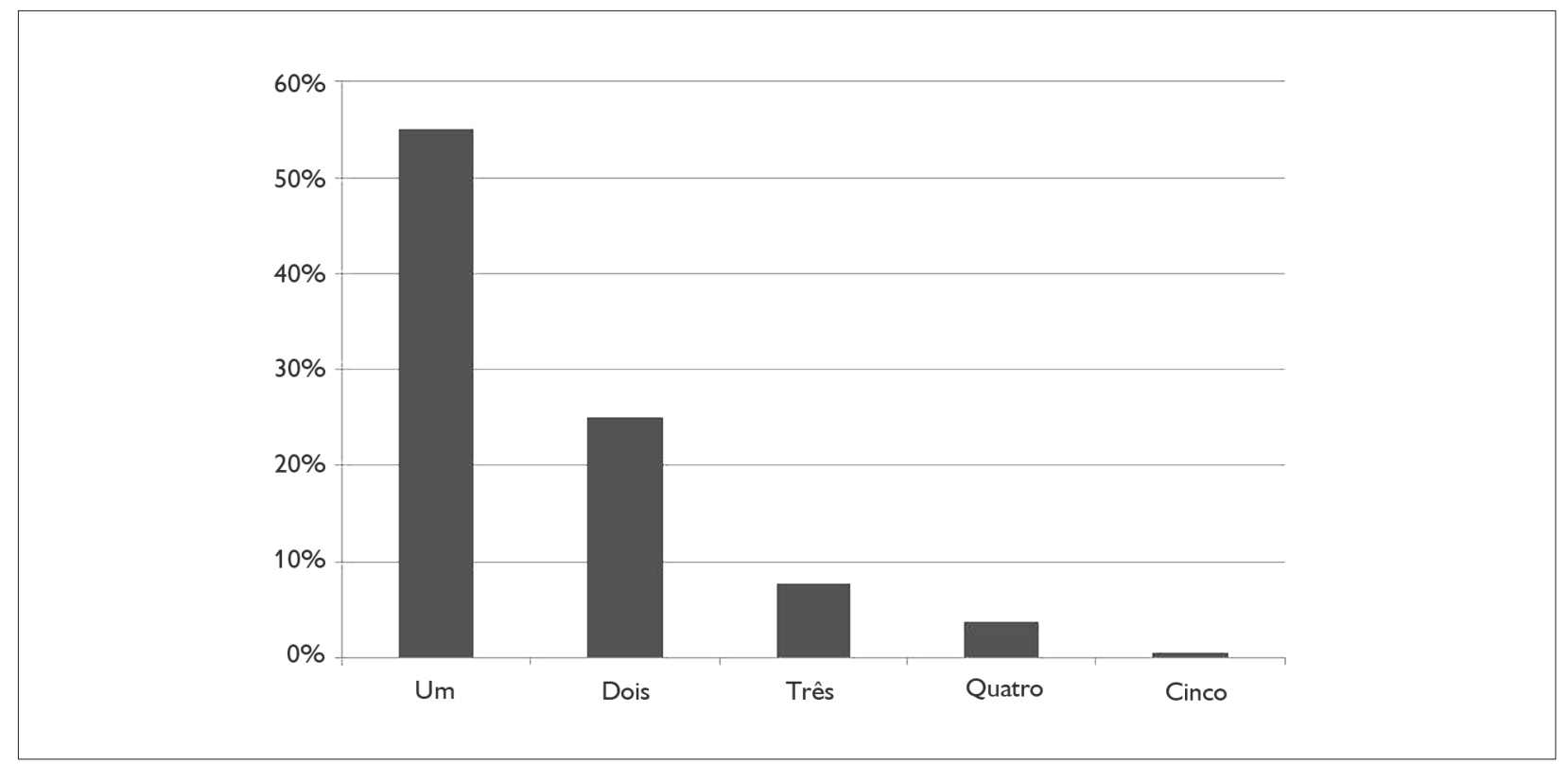

Figura 2. Percentagem de espécies, que ocorrem em um tipo de substrato, ou simultaneamente em dois a cinco substratos distintos encontrados no Parque Estadual Intervales (PEI), São Paulo.

caracteriza ambientes em regeneração, quando a maioria das espécies ocorre em vários tipos de substratos, e ambientes estabilizados, quando são mais restritas a um tipo de substrato. Como relacionado anteriormente, - PEI apresenta Mata Atlântica de encosta pouco alterada pela intervenção humana e consegue conciliar o ecoturismo com a preservação ambiental (Zipparro et al., 2005; Sarfati \& Sano, 2012).

Plantas generalistas (108 espécies, 49\%) são mais comuns do que plantas típicas de sombra (73 espécies, 33\%) e plantas típicas de sol (34 espécies, 16\%). Dados sobre o grupo ecológico são desconhecidos para os musgos Cryphaea brevipila, Daltonia marginata, Rhaphidostegium cylindrothecium, Sematophyllum leucostomum, e para a hepática Cololejeunea vitalana (cinco espécies, 2\%, Apêndice 1). Espécies generalistas ocorrem tanto em locais úmidos e sombrios, quanto em locais mais secos e expostos à alta intensidade luminosa (Santos \& Costa, 2008) e, portanto, são as menos afetadas pela perda do habitat, persistindo em locais perturbados (Alvarenga et al., 2010). Como já citado, o PEI situa-se em uma área perturbada pela mineração, pela própria ocupação e agricultura, mas sem concentrações urbanas significativas, e apresenta Mata Atlântica de encosta, com pouca intervenção antrópica (Zipparro et al., 2005; Sallun Filho et al., 2010). Predomínio de espécies generalistas e menor ocorrência de espécies típicas de sombra e de sol já foram relatados para outras formações florestais da Mata Atlântica, como floresta ombrófila aberta e restinga (Silva \& Pôrto, 2010; Santos et al., 2011), para áreas antrópicas (Visnadi, 2013a) e para o PETAR, circunvizinho do PEI, também com floresta ombrófila densa sobre relevo cárstico (Ivanauskas et al., 2012; Visnadi, 2013b).

Formas de vida associadas a ambientes de maior umidade (Santos \& Costa, 2008; Santos et al., 2011; Glime, 2013) são mais comuns na Mata Atlântica do PEI (pendente - 8\%, flabelado - 7\%, dendroide - 5\%, trama - 5\%) em comparação com as áreas abertas de Ubatuba (pendente - 2,7\%, flabelado - 2,7\%, dendroide - 0,9\%, trama - 0,9\%; Visnadi, 2013a), exceto duas formas de vida, que são mais comuns nas últimas áreas (tapete $60 \%$, talosa - 11\%) do que no PEI (tapete - 49\%, talosa - 5\%). As formas de vida relacionadas a ambientes com 
baixa umidade, segundo os três primeiros trabalhos, foram registradas apenas para $20 \%$ das espécies no local (tufo - 19,5\%; coxim - 0,5\%, Syzygiella rubricaulis). Dados sobre forma de vida são desconhecidos para Cryphaea brevipila e Daltonia marginata (1\%, Apêndice 1).

PEI e PETAR são parques circunvizinhos, que se situam em regiões cársticas, com floresta ombrófila densa sobre o relevo montanhoso da serra de Paranapiacaba (Ivanauskas et al., 2012). PETAR abrange parte dos municípios de Guapiara, Iporanga e Apiaí (Visnadi, 2013b) e o PEI também, além dos municípios de Eldorado Paulista, Ribeirão Grande, Sete Barras e Capão Bonito, como relacionado anteriormente. Todavia, os rótulos do material estudado nem sempre especificam em quais municípios as coletas foram realizadas para cada parque. Comparando a brioflora de ambas as unidades de conservação, verificou-se que PETAR e PEI apresentam brioflora pouco semelhante, com apenas 32\% de espécies em comum (70 das 220 espécies do PEI; índice de similaridade de Sørensen $=0,43 \%$ ). Além disso, a brioflora do PETAR, com 109 espécies, foi registrada em 259 exsicatas (Visnadi, 2013b), enquanto que a brioflora do PEI, com 220 espécies, em 767 exsicatas de material estudado.

A brioflora do PEI (220 espécies) compõe-se de maior número de espécies do que a brioflora registrada, até o momento, para a Mata Atlântica de Mogi das Cruzes (216 espécies), Iguape (176 espécies), São Sebastião (70 espécies), Bertioga (65 espécies) e Caraguatatuba (31 espécies), porém com menor número do que a flora de briófitas até então listada para esse mesmo ecossistema em Santo André (239 espécies) e Ubatuba (384 espécies), no estado de São Paulo (Visnadi, 2005, 2012; Peralta \& Yano, 2008, 2012; Yano et al., 2009, Santos et al., 2011; Yano, 2013).

Quanto mais rica em espécies de briófitas é a Mata Atlântica de outras localidades paulistas, maior é a semelhança entre a brioflora dessas localidades e a do PEI. Portanto, segundo os dados disponíveis até o momento, a flora de briófitas do parque estudado, com
220 espécies, assemelha-se mais com a flora de Ubatuba (384 espécies) e menos com a flora de Mogi das Cruzes (216 espécies), Iguape (176 espécies), São Sebastião (70 espécies), Bertioga (65 espécies) e Caraguatatuba (31 espécies) (Figura 3).

A flora de briófitas de Santo André (239 espécies) assemelha-se mais à flora do PEI (220 espécies), do que Iguape (176 espécies), São Sebastião (70 espécies), Bertioga (65 espécies) e Caraguatatuba (31 espécies). Todavia, em Santo André, a brioflora possui várias espécies ameaçadas de extinção em São Paulo, e com distribuição geográfica disjunta entre esse local e outros estados brasileiros (Yano et al., 2009), tornando-a mais distinta da brioflora do PEI (220 espécies) do que Mogi das Cruzes (216 espécies).

O PEI apresenta 53 espécies (24\%), que são frequentes na Mata Atlântica paulista, pois ocorrem em cinco a oito localidades, incluindo a área de estudo (Ubatuba, Caraguatatuba, São Sebastião, Bertioga, Mogi das Cruzes, Santo André, Iguape e PEI). Lejeunea flava é a espécie mais comum, pois ocorre em todas as localidades relacionadas anteriormente; a maioria das espécies (130 espécies, 59\%) é menos frequente nesse ecossistema em São Paulo, pois se distribui em duas a quatro localidades paulistas, já citadas, incluindo a área de estudo (Visnadi, 2005, 2012; Peralta \& Yano, 2008, 2012; Yano et al., 2009; Santos et al., 2011; Yano, 2013). Esses dados evidenciam que a brioflora do PEl é característica da Mata Atlântica em São Paulo, pois $83 \%$ das espécies ocorrem também nesse ecossistema de outras localidades no estado.

A brioflora do PEI, contudo, é também peculiar, por se distinguir da brioflora destes sete municípios, relacionados anteriormente, pela ocorrência de 37 espécies (17\%), que são exclusivas do PEI, não ocorrendo na Mata Atlântica de outra localidade paulista; dessas, 32 espécies são citadas pela primeira vez para a Mata Atlântica do estado de São Paulo e cinco (Barbula indica, Cryphaea brevipila, Isopterygium affusum, Schlotheimia appressifolia, Cololejeunea vitalana) já foram registradas na literatura para o parque (Apêndice 1). 


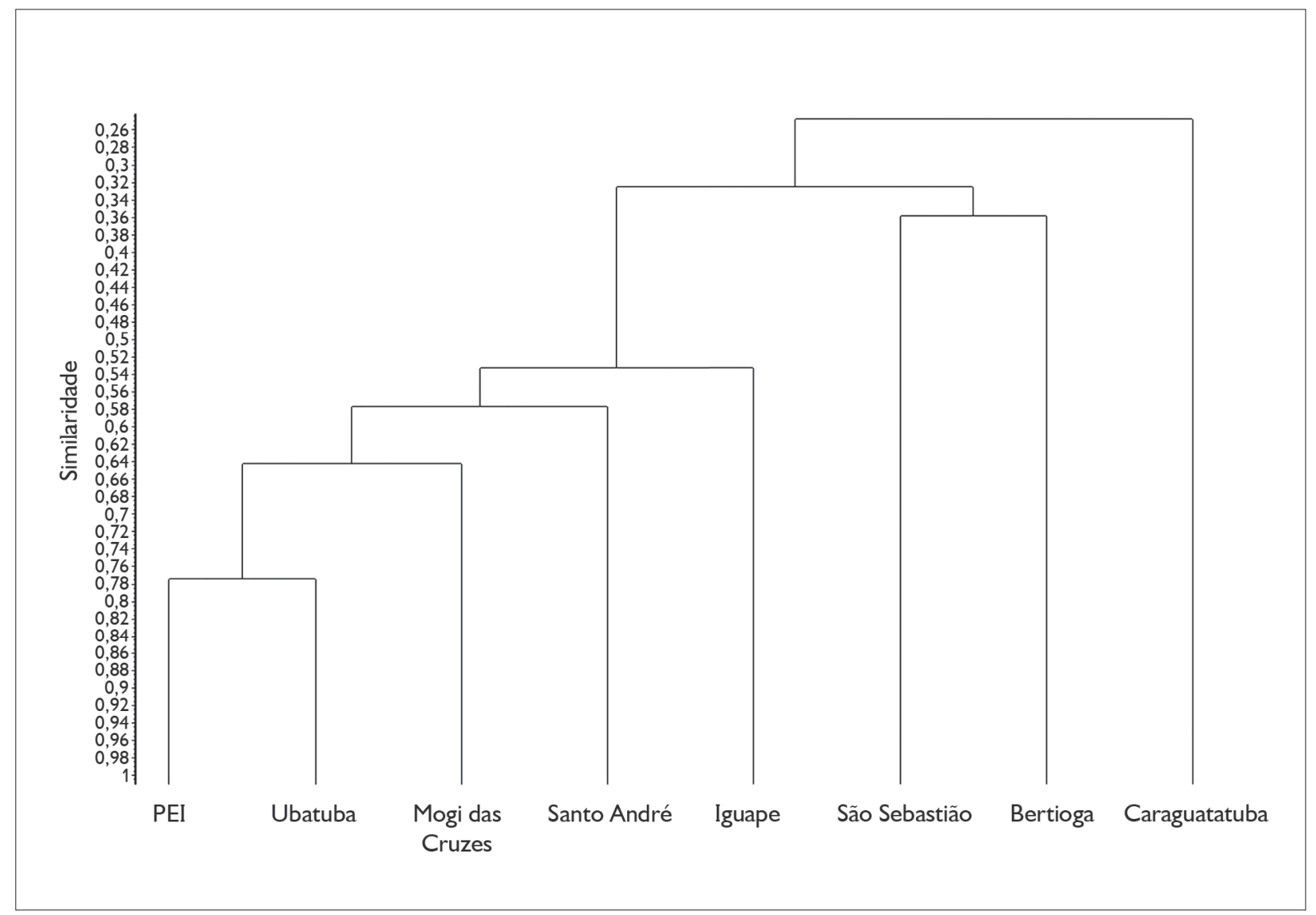

Figura 3. Agrupamento de sete localidades paulistas e do Parque Estadual Intervales (PEI), dos ecossistemas em relação à presença das espécies de briófitas na Mata Atlântica; correlação cofenética $=0,9554$. A exclusão das espécies restritas (que ocorrem apenas no PEI) evidencia o mesmo padrão de distribuição das briófitas, mas com maior similaridade entre os locais (não ilustrado); correlação cofenética $=0,9665$.

Em São Paulo, briófitas neotropicais são a maioria das espécies nos ecossistemas da Estação Ecológica Jureia-Itatins (Visnadi, 2012) e na Mata Atlântica de Intervales (113 espécies, 51\%), na qual sete espécies também alcançam a América subtropical, ou a Índia, e cinco são primeiras citações para o Brasil (Leskeodon andicola) e para São Paulo (Macromitrium longifolium, Orthostichopsis praetermissa, Pelekium scabrosulum e Thamniopsis cruegeriana); essas últimas espécies encontram-se ameaçadas no estado paulista, categoria vulnerável (VU).

Espécies pantropicais são menos numerosas do que espécies neotropicais (Tan \& Pócs, 2000), como no PEI (27 espécies, 12\%), em que duas espécies também se estendem até as regiões temperadas e oceânicas do hemisfério norte. Disjunções de espécies são comuns entre a região neotropical e a África (Shaw, 2009), mas, na área de estudo, espécies afro-americanas são pouco frequentes (21 espécies, 10\%) e, entre elas, três também alcançam o oeste da Europa, ou a Índia. Poucas espécies de briófitas são cosmopolitas (Gignac, 2001), inclusive no PEI, no qual totalizam 20 espécies (9\%), incluindo uma subcosmopolita. Esses dados são similares aos resultados encontrados para florestas tropicais de outras localidades brasileiras (Costa \& Lima, 2005; Santos \& Costa, 2008).

A brioflora do PEI também apresenta espécies endêmicas do Brasil (13 espécies, 6\%) e espécies que 
ocorrem no país, mas que também se estendem a outros países da América do Sul, até o Caribe, a América Central, a América do Norte, as regiões temperadas do hemisfério norte ou a Índia (11 espécies, 5\%), entre as quais Fissidens dendrophilus (Brasil e Guiana Francesa) é citação nova para São Paulo, e Chryso-hypnum squarrosulum (México e América Central) é citação nova para o Brasil; ambas as espécies encontram-se ameaçadas no estado paulista, categoria vulnerável (VU).

Poucas espécies distribuem-se na América do Sul (9 espécies, 4\%) e, entre elas, duas também alcançam a Jamaica e o México, ou a Austrália e a Nova Zelândia; menos espécies ainda ocorrem em todo o continente americano (5 espécies, 2,5\%), algumas delas também frequentes no Caribe, Japão ou na Coreia. Finalmente, Eurhynchium hians (0,5\% das espécies), que no Brasil se restringe a São Paulo, mas que também ocorre no Haiti, na América do Norte, Europa, Ásia e África, não se encontra ameaçada no estado paulista (least concern - LC).

A brioflora do PEI distribui-se amplamente pelas regiões do Brasil (Sudeste - 100\%, Sul - 86\%, Nordeste - 77\%, Norte - 60\% e Centro-Oeste - 56\%), pelos estados do Sudeste (São Paulo - 100\%, Rio de Janeiro - 92\%, Minas Gerais - 85\%, Espírito Santo - 66\%) e da região Sul (Santa Catarina - 72\%, Rio Grande do Sul - 67\%, Paraná - 66\%), na Bahia (68\%) e em Pernambuco (57\%), pois apresentam mais da metade das espécies listadas.

\section{CONCLUSÃO}

A ocorrência no PEI de espécies inéditas para o Brasil, de espécies endêmicas do país e de espécie que no Brasil se restringe ao estado de São Paulo demonstra a importância do parque na conservação e na proteção da diversidade da brioflora brasileira. As novas ocorrências de espécies para São Paulo, que se encontram ameaçadas no estado, evidenciam que o PEI também contribui para a conservação e a proteção da flora de briófitas paulistas. A riqueza da brioflora do parque enfatiza a importância do PEI na conservação e na proteção da flora de briófitas da Mata Atlântica local.

\section{AGRADECIMENTOS}

À S. F. B. Trufem, pela organização das viagens de campo, e a D. M. Vital, pela ajuda na coleta e identificação das briófitas, ambos então do Instituto de Botânica de São Paulo; à Direção do Parque Estadual Intervales (PEI), pelo apoio logístico na área de estudo; e à Fundação para a Conservação e a Produção Florestal do Estado de São Paulo - Fundação Florestal, pela permissão da coleta do material botânico no Parque.

\section{REFERÊNCIAS}

ALLEN, B. H., 1994. Moss flora of Central America. Part 1. Sphagnaceae-Calymperaceae. Missouri Botanical Garden, Monographs in Systematic Botany 49: 1-242.

ALLEN, B. H., 2002. Moss flora of Central America. Part 2. Encalyptaceae-Orthotrichaceae. Missouri Botanical Garden, Monographs in Systematic Botany 90: 1-699.

ALLEN, B. H., 2010. Moss flora of Central America. Part 3. Anomodontaceae-Symphyodontaceae. Missouri Botanical Garden, Monographs in Systematic Botany 117: 1-731.

ALLEN, B. H. \& R. E. MAGILL, 2003. A revision of Pilotrichella (Lembophyllaceae: Musci). Acta Academiae Paedagogicae Agriensis, Sectio Biologiae 24: 43-83.

ALLEN, B. H. \& R. E. MAGILL, 2007. A revision of Orthostichella (Neckeraceae). The Bryologist 110(1): 1-45.

ALVARENGA, L. D. P., K. C. PÔRTO \& J. R. P. M. OLIVEIRA, 2010. Habitat loss effects on spatial distribution of non-vascular epiphytes in a Brazilian Atlantic Forest. Biodiversity and Conservation 19(3): 619-635.

BATES, J. W., 2009. Mineral nutrition and substratum ecology. In: B. GOFFINET \& A. J. SHAW (Eds.): Bryophyte biology: 2. ed.: 299-356. Cambridge University Press, New York.

BORDIN, J. \& O. YANO, 2013. Fissidentaceae (Bryophyta) do Brasil. Boletim do Instituto de Botânica 22: 1-169.

BUCK, W. R., 1998. Pleurocarpous mosses of the West Indies. Memoirs of the New York Botanical Garden 82: 1-400.

BUCK, W. R. \& D. M. VITAL, 1992. Paranapiacabaea paulista, a new genus and species of Sematophyllaceae from southeastern Brazil. Brittonia 44(3): 339-343.

COSTA, D. P. \& F. M. LIMA, 2005. Moss diversity in the tropical rainforests of Rio de Janeiro, southeastern Brazil. Revista Brasileira de Botânica 28(4): 671-685. 
COSTA, D. P., K. C. PÔRTO, A. P. LUIZI-PONZO, A. L. ILKIUBORGES, C. J. P. BASTOS, P. E. A. S. CÂMARA, D. F. PERALTA, S. B. V. BÔAS-BASTOS, C. A. A. IMBASSAHY, D. K. HENRIQUES, H. C. S. GOMES, L. M. ROCHA, N. D. SANTOS, T. S. SIVIERO, T. F. VAZ-IMBASSAHY \& S. P. CHURCHILL, 2011. Synopsis of the Brazilian moss flora: checklist, distribution and conservation. Nova Hedwigia 93(3-4): 277-334.

COSTA, D. P. \& D. F. PERALTA, 2014. Briófitas. In: JARDIM BOTÂNICO DO RIO DE JANEIRO. Lista de espécies da Flora do Brasil. Disponível em: <http://floradobrasil.jbrj.gov.br/jabot/ floradobrasil/FB128472>. Acesso em: 19 agosto 2014.

CRANDALL-STOTLER, B., R. E. STOTLER \& D. G. LONG, 2009. Morphology and classification of the Marchantiophyta. In: B. GOFFINET \& A. J. SHAW (Eds.): Bryophyte biology: 2. ed.: 1-54. Cambridge University Press, New York.

CRUM, H., 1992. Miscellaneous notes on the genus Sphagnum. 3. New species from Brazil. The Bryologist 95(4): 419-429.

CRUM, H. A. \& L. E. ANDERSON, 1981. Mosses of Eastern North America: 1-1328. Columbia University Press, New York.

FLORA OF NORTH AMERICA EDITORIAL COMMITTEE, 2007. Flora of North America: 1-713. Oxford University Press (Bryophytes: Mosses: v. 27, Bryophyta, part 1), New York.

FLORSCHÜTZ-DEWAARD, J., 1996. Sematophyllaceae. In: A. R. A. GÖRTS-VAN RIJN (Ed.): Flora of the Guianas: Series C: Bryophytes, Fascicle 1. Musci III: 384-438. Royal Botanic Gardens, Kew.

FRAHM, J.-P., 1991. Dicranaceae: Campylopodioideae, Paraleucobryoideae. Flora Neotropica 54: 1-238.

GIGNAC, L. D., 2001. Bryophytes as indicators of climate change (invited essay, new frontiers in bryology and lichenology). The Bryologist 104(3): 410-420.

GLIME, J. M., 2013. Adaptative strategies: growth and life forms. In: J. M. GLIME (Ed.): Bryophyte ecology: v. 1: Physiological Ecology. Michigan Technological University and the International Association of Bryologists, Michigan. Disponível em: < http://www. bryoecol.mtu.edu/chapters/4-5AdaptStratForm.pdf > . Acesso em: 8 setembro 2014.

GOFFINET, B., W. R. BUCK \& A. J. SHAW, 2009. Morphology, anatomy, and classification of the Bryophyta. In: B. GOFFINET \& A. J. SHAW (Eds.): Bryophyte biology: 2. ed.: 55-138. Cambridge University Press, New York.

GRADSTEIN, S. R., S. T. CHURCHILL \& N. SALAZAR-ALLEN, 2001. Guide to the bryophytes of Tropical America. Memoirs of the New York Botanical Garden 86: 1-577.

GRADSTEIN, S. R. \& D. P. COSTA, 2003. The Hepaticae and Anthocerotae of Brazil. Memoirs of the New York Botanical Garden 87: 1-318.
HELL, K., 1969. Briófitas talosas dos arredores da cidade de São Paulo (Brasil). Boletim da Faculdade de Filosofia e Ciências da Universidade de São Paulo, Série Botânica 335(25): 1-187.

INDEX HEPATICARUM, [s. d.]. Conservatoire et Jardin Botaniques de La Ville de Genève. Disponível em: < http://www. ville-ge.ch/musinfo/bd/cjb/hepatic/>. Acesso em: 21 agosto 2014.

INTERNATIONAL UNION FOR CONSERVATION OF NATURE AND NATURAL RESOURCES (IUCN), [s. d.]. The IUCN red list of threatened species. Disponível em: <http://www.iucnredlist. org/>. Acesso em: 21 agosto 2014

IRELAND, R. R., 1992. The moss genus Isopterygium (Hypnaceae) in Latin America. Tropical Bryology 6: 111-132.

IVANAUSKAS, N. M., R. L. MIASHIKE, I. R. L. GODOY, F. M. SOUZA, M. M. KANASHIRO, I. F. A. MATTOS, M. T. Z. TONIATO \& G. A. D. C. FRANCO, 2012. A vegetação do Parque Estadual Turístico do Alto Ribeira (PETAR), São Paulo, Brasil. Biota Neotropica 12(1): 147-177

KRUIJER, H., 2002. Hypopterygiaceae of the World. Blumea: Journal of Plant Taxonomy and Plant Geography Suplement 13: 1-388.

MACHADO, P. S. \& A. P. LUIZI-PONZO, 2011. Urban bryophytes from Southeastern Brazilian area (Juiz de Fora, Minas Gerais, Brazil). Boletim do Instituto de Botânica 21: 223-261.

MAGILL, R. E. \& J. VAN ROOY, 1998. Flora of Southern Africa. Bryophyta: Erpodiaceae - Hookeriaceae: 445-662. National Botanical Institute (Part 1 Musci, n. 3), Pretoria.

MARTINELLI, G. \& M. A. MORAES (Orgs.), 2013. Livro vermelho da flora do Brasil: 1. ed.: 1-1100. Instituto de Pesquisas Jardim Botânico do Rio de Janeiro, Centro Nacional de Conservação da Flora - CNCFLORA, Rio de Janeiro.

MEAGHER, D. \& B. FUHRER, 2003. A field guide to the mosses \& allied plants of Southern Australia: 1-280. Australian Biological Resources Study e Field Naturalists Club of Victoria (Flora of Australia Supplementary Series, 20), Canberra.

NISHIMURA, N. \&H. ANDO, 1986. A revision of some Mittenothamnium species described from Mexico. The Bryologist 89(1): 66-69.

OLIVEIRA-E-SILVA, M. I. M. N. \& O. YANO, 2000. Musgos de Mangaratiba e Angra dos Reis, Rio de Janeiro. Brasil. Boletim do Instituto de Botânica 14: 1-137.

PEPINELLI, M., S. TRIVINHO-STRIXINO \& N. HAMADA, 2005. Imaturos de Simuliidae (Diptera, Nematocera) e caracterização de seus criadouros no Parque Estadual Intervales, SP, Brasil. Revista Brasileira de Entomologia 49(4): 527-530.

PERALTA, D. F. \& O. YANO, 2008. Briófitas do Parque Estadual da Ilha Anchieta, Ubatuba, estado de São Paulo, Brasil. Iheringia, Série Botânica 63(1): 101-127. 
PERALTA, D. F. \& O. YANO, 2011. Checklist of bryophytes (Antocerotophyta, Bryophyta e Marchantiophyta) from São Paulo State. Biota Neotropica 11(1a). Disponível em: <http://www. biotaneotropica.org.br/v11n1a/en/abstract?inventory + bn011110 1a2011>. Acesso em: 17 setembro 2014.

PERALTA, D. F. \& O. YANO, 2012. Briófitas da Serra do Itapeti. In: M. S. C. MORINI \& V. F. O. MIRANDA (Eds.): Serra do Itapeti. Aspectos históricos, sociais e naturalísticos: 75-86. Canal 6, Bauru.

PORLEY, R., 2008. Arable bryophytes: a field guide to the mosses, liverworts and hornworts of cultivated land in Britain and Ireland: 1-140. Wildguides, Hampshire.

REESE, W. D., 1993. Calymperaceae. Flora Neotropica 58: 1-102.

RENZAGLIA, K. S., J. C. VILLARREAL \& R. J. DUFF, 2009. New insights into morphology, anatomy, and systematics of hornworts. In: B. GOFFINET \& A. J. SHAW (Eds.): Bryophyte biology: 2. ed.: 139-171. Cambridge University Press, New York.

RICHARDS, P. W., 1984. The ecology of tropical forest bryophytes. In: R. M. SCHUSTER (Ed.): New manual of bryology: 2. v.: 12331270. Hattori Botanical Laboratory, Nichinan.

RODRIGUES, R. R., C. A. JOLY, M. C. W. BRITO, A. PAESE, J. P. METZGER, L. CASATTI, M. A. NALON, N. MENEZES, N. M. IVANAUSKAS, V. BOLZANI \& V. L. R. BONONI, 2008. Diretrizes para a conservação e restauração da biodiversidade no Estado de São Paulo: 1-248. Instituto de Botânica/Imprensa Oficial do Estado de São Paulo, São Paulo.

SALLUN FILHO, W., J. A. FERRARI, S. T. HIRUMA, A. E. M. SALLUN \& I. KARMANN, 2010. O carste no plano de manejo do Parque Estadual Intervales e zona de amortecimento, Estado de São Paulo, Brasil. Revista Escola de Minas 63(3): 441-448.

SANTOS, N. D. \& D. P. COSTA, 2008. A importância de Reservas Particulares do Patrimônio Natural para a conservação da brioflora da Mata Atlântica: um estudo em El Nagual, Magé, RJ, Brasil. Acta Botanica Brasilica 22(2): 359-372.

SANTOS, N. D., D. P. COSTA, L. S. KINOSHITA \& G. J. SHEPHERD, 2011. Aspectos brioflorísticos e fitogeográficos de duas formações costeiras de Floresta Atlântica da Serra do Mar, Ubatuba/ SP, Brasil. Biota Neotropica 11(2): 425-438.

SARFATI, G. \& N. N. SANO, 2012. Estudo comparado da gestão das visitações nos Parques Estaduais Turísticos do Alto Ribeira (PETAR) e Intervales (PEI). Turismo em Análise 23(1): 207-237.

SCHÄFER-VERWIMP, A., 1991. Contribution to the knowledge of the bryophyte flora of Espírito Santo, Brazil. The Journal of the Hattori Botanical Laboratory 69: 147-170.

SCHÄFER-VERWIMP, A., 1992. New or interesting records of Brazilian bryophytes, III. The Journal of the Hattori Botanical Laboratory 71: 55-68.
SHARP, A. J., H. CRUM \& P. M. ECKEL, 1994. The moss flora of Mexico. Memoirs of the New York Botanical Garden 69(1-2): 1-1113.

SHAW, A. J., 2009. Bryophyte species and speciation. In: B. GOFFINET \& A. J. SHAW (Eds.): Bryophyte biology: 2. ed.: 445485. Cambridge University Press, New York.

SHEPHERD, G. J., 2009. Fitopac versão 2.1. Universidade Estadual de Campinas, Campinas.

SILVA, M. P. P. \& K. C. PÔRTO, 2010. Spatial structure of bryophyte communities along an edge-interior gradient in an Atlantic Forest remnant in Northeast Brazil. Journal of Bryology 32(2): 101-112.

SIM, T. R., 1973. The bryophyta of South Africa: 1-475. Otto Koeltz Antiquariat, Koenigstein.

SISTEMA NACIONAL DE UNIDADES DE CONSERVAÇÃO (SNUC), 2004. Texto da Lei 9.985 de 18 de julho de 2000 e vetos da presidência da República ao PL aprovado pelo Congresso Nacional e Decreto $N^{\circ} 4.340$, de 22 de agosto de 2002: 1-76. Conselho Nacional da Reserva da Biosfera da Mata Atlântica (Cadernos da Reserva da Biosfera da Mata Atlântica: série conservação e áreas protegidas, 18), São Paulo.

TAN, B. C. \& T. PÓCS, 2000. Bryogeography and conservation of bryophytes. In: A. J. SHAW \& B. GOFFINET (Eds.): Bryophyte biology: 403-448. Cambridge University Press, Cambridge.

TROPICOS, [s. d.]. Tropicos.org. Missouri Botanical Garden, Saint Louis. Disponível em: <http://www.tropicos.org/>. Acesso em: 14 agosto 2014.

VAlENTE, E. B., K. C. PÔRTO \& C. J. P. BASTOS, $2013 \mathrm{a}$ Species richness and distribution of bryophytes within different phytophysiognomies in the Chapada Diamantina region of Brazil. Acta Botanica Brasilica 27(2): 294-310

VALENTE, E. B., K. C. PÔRTO, C. J. P. BASTOS \& J. BALLEJOSLOYOLA, 2013b. Diversity and distribution of the bryophyte flora in montane forests in the Chapada Diamantina region of Brazil. Acta Botanica Brasilica 27(3): 506-518.

VISNADI, S. R., 2002. Meteoriaceae (Bryophyta) da Mata Atlântica do estado de São Paulo. Hoehnea 29(3): 159-187.

VISNADI, S. R., 2005. Brioflora da Mata Atlântica do estado de São Paulo: região norte. Hoehnea 32(2): 215-231.

VISNADI, S. R., 2006. Sematophyllaceae da Mata Atlântica do nordeste do Estado de São Paulo. Hoehnea 33(4): 455-484.

VISNADI, S. R., 2008. Marchantiophyta e Bryophyta de manguezais do estado de São Paulo, Brasil. Boletim do Museu Paraense Emílio Goeldi. Ciências Naturais 3(1): 69-80.

VISNADI, S. R., 2009. Briófitas do caxetal, em Ubatuba, São Paulo, Brasil. Tropical Bryology 30: 8-14.

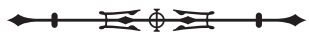


VISNADI, S. R., 2010. Briófitas das formações florestais não inundáveis das restingas do estado de São Paulo, Brasil. Tropical Bryology 32: 74-86.

VISNADI, S. R., 2011. Briófitas dos picos do Cuscuzeiro e do Cardoso, estado de São Paulo, Brasil. Boletim do Museu Paraense Emílio Goeldi. Ciências Naturais 6(3): 307-317.

VISNADI, S. R., 2012. Briófitas da Estação Ecológica Jureia-Itatins, estado de São Paulo, Brasil. Tropical Bryology 34: 17-31.

VISNADI, S. R., 2013a. Briófitas de áreas antrópicas do Parque Estadual da Serra do Mar, Núcleo Picinguaba, Ubatuba, estado de São Paulo, Brasil. Boletim do Museu Paraense Emílio Goeldi. Ciências Naturais 8(1): 49-62.

VISNADI, S. R., 2013b. Brioflora do Parque Estadual Turístico do Alto do Ribeira (Petar), estado de São Paulo, Brasil. Tropical Bryology 35: 52-63.

VISNADI, S. R. \& D. M. VITAL, 1997. Bryophytes from greenhouses of the Institute of Botany, São Paulo, Brazil. Lindbergia 22(1): 44-46.

VISNADI, S. R. \& D. M. VITAL, 2000. Lista das briófitas ocorrentes no Parque Estadual das Fontes do Ipiranga, PEFI. Hoehnea 27(3): 279-294.

WIGGINTON, M. J., 2004. E. W. Jones's liverwort and hornwort flora of West Africa: 1-443. National Botanic Garden of Belgium (Scripta Botanica Belgica, 30), Meise.

YAMAGUCHI, T., 1993. A revision of the genus Leucobryum (Musci) in Asia. The Journal of the Hattori Botanical Laboratory 73: 1-123.
YANO, O., 2008. Catálogo de antóceros e hepáticas brasileiros: literatura original, basiônimo, localidade-tipo e distribuição geográfica. Boletim do Instituto de Botânica 19: 1-110.

YANO, O., 2010. Levantamento de novas ocorrências de briófitas brasileiras. Instituto de Botânica, São Paulo. Disponível em: <http://botanica.sp.gov.br/files/2013/09/virtuais_1briofitas. pdf > . Acesso em: 30 junho 2014.

YANO, O., 2011. Catálogo de musgos brasileiros: literatura original, basiônimo, localidade-tipo e distribuição geográfica. Instituto de Botânica, São Paulo. Disponível em: < http://botanica. sp.gov.br/files/2013/09/virtuais_2musgos.pdf > . Acesso em: 30 junho 2014.

YANO, O., 2013. Adição do levantamento de novas ocorrências de briófitas brasileiras (2009-2011). Instituto de Botânica, São Paulo. Disponível em: <http://botanica.sp.gov.br/files/2011/11/ adicao_de_briofitas.pdf >. Acesso em: 30 junho 2014.

YANO, O., S. R. VISNADI \& D. F. PERALTA, 2009. Briófitas. In: M. I. M. S. LOPES, M. KIRIZAWA \& M. M. R. F. MELO (Eds.): Patrimônio da Reserva Biológica do Alto da Serra de Paranapiacaba: a antiga Estação Biológica do Alto da Serra: 255267. Imprensa Oficial do Estado de São Paulo, São Paulo.

ZIPPARRO, V. B., F. A. G. GUILHERME, R. J. ALMEIDA-SCABBIA \& L. P. C. MORELLATO, 2005. Levantamento florístico de Floresta Atlântica no sul do Estado de São Paulo, Parque Estadual Intervales, Base Saibadela. Biota Neotropica 5(1): 127-144. 
APÊNDICE 1. Briófitas ocorrentes em remanescente da Mata Atlântica (floresta ombrófila densa) sobre regiões cársticas do Parque Estadual Intervales (PEI). Legendas: Fonte - 1 = Buck \& Vital (1992), 2 = Crum (1992), 3 = Schäfer-Verwimp (1992), $4=$ Visnadi (2002), 5 = Gradstein \& Costa (2003), 6 = Yano (2010), 7 = Yano (2011), 8 = Yano (2013), X = material coletado e depositado no Herbário (SP); Substrato - ALV = parede de alvenaria, CFV = casca de forófito vivo, CFM = casca de forófito morto, ES = estalactite, $\mathrm{FO}=$ folhas, $\mathrm{NP}=$ ninho de pássaro, $\mathrm{RO}=$ rocha, $\mathrm{SO}=$ solo, TE $=$ telhado; Grupo ecológico - gen $=$ generalista sol = típica de sol, som = típica de sombra; Forma de vida $-C=$ coxim, $D=$ dendroide, $F=$ flabelado, $P=$ pendente, TF $=$ tufo, $\mathrm{TL}=$ talosa, $\mathrm{TP}=$ tapete, $\mathrm{TR}=$ trama; ${ }^{*}=$ citação nova para a Mata Atlântica paulista; s/n = sem número de coletor; d.l. $=$ dados da literatura.

\begin{tabular}{|c|c|c|c|c|c|}
\hline Espécies & Fonte & Substrato & $\begin{array}{l}\text { Grupo } \\
\text { ecológico }\end{array}$ & $\begin{array}{l}\text { Forma de } \\
\text { vida }\end{array}$ & Voucher \\
\hline \multicolumn{6}{|l|}{ ANTHOCEROTOPHYTA } \\
\hline \multicolumn{6}{|l|}{ Dendrocerotaceae } \\
\hline Dendroceros crispus (Sw.) Nees & $x$ & CFV & gen & $\mathrm{TL}$ & Visnadi \& Vital 4977 \\
\hline \multicolumn{6}{|l|}{ Notothyladaceae } \\
\hline Phaeoceros laevis (L.) Prosk. & $x$ & SO & gen & $\mathrm{TL}$ & Vital s/n (SP387688) \\
\hline Phaeoceros laevis subsp. carolinianus (Michx.) Prosk. & $x$ & $\mathrm{SO}$ & gen & $\mathrm{TL}$ & Visnadi \& Vital 4986 \\
\hline \multicolumn{6}{|l|}{ BRYOPHYTA } \\
\hline \multicolumn{6}{|l|}{ Bartramiaceae } \\
\hline Leiomela bartramioides (Hook.) Paris & $x$ & CFM, CFV & gen & TF & Visnadi \& Vital 4776 \\
\hline Philonotis uncinata (Schwägr.) Brid. & $x$ & $\mathrm{SO}$ & gen & $\mathrm{TF}$ & Visandi \& Vital 4691 \\
\hline \multicolumn{6}{|l|}{ Brachytheciaceae } \\
\hline Aerolindigia capillacea (Hornsch.) M. Menzel & $x$ & - & som & $P$ & Vital \& Buck 20558 \\
\hline Brachythecium ruderale (Brid.) W.R. Buck & $x$ & SO & sol & $\mathrm{TP}$ & Visnadi \& Vital 4780 \\
\hline Eurhynchium hians (Hedw.) Sande Lac. & $x$ & $\mathrm{SO}$ & som & $\mathrm{TP}$ & Visnadi \& Vital 4781 \\
\hline Helicodontium capillare (Hedw.) A. Jaeger & $x$ & CFM, CFV & sol & $\mathrm{TR}$ & Visnadi \& Vital 4943 \\
\hline Meteoridium remotifolium (Müll. Hal.) Manuel & $4, x$ & ALV, CFV, FO, SO & gen & $\mathrm{P}$ & Visnadi \& Vital 4746 \\
\hline *Rhynchostegium serrulatum (Hedw.) A. Jaeger. & $x$ & CFM, RO, SO & gen & $\mathrm{TP}$ & Visnadi \& Vital 4881 \\
\hline Squamidium brasiliense (Hornsch.) Broth. & $4, X$ & CFV & som & P & Visnadi \& Vital 5072 \\
\hline Squamidium leucotrichum (Taylor) Broth. & $x$ & CFV & gen & $\mathrm{P}$ & Visnadi \& Vital 4816 \\
\hline Zelometeorium ambiguum (Hornsch.) Manuel & $4, \times$ & CFV, RO & gen & $\mathrm{P}$ & Visnadi \& Vital 4848 \\
\hline Zelometeorium patens (Hook.) Manuel & $4, \times$ & CFV & gen & $\mathrm{P}$ & Visnadi \& Vital 4788 \\
\hline Zelometeorium patulum (Hedw.) Manuel & $4, X$ & CFV, FO, NP & gen & $\mathrm{P}$ & Visnadi \& Vital 5071 \\
\hline \multicolumn{6}{|l|}{ Bruchiaceae } \\
\hline Trematodon longicollis Michx. & $x$ & - & sol & TF & Vital \& Buck 20441 \\
\hline \multicolumn{6}{|l|}{ Bryaceae } \\
\hline Bryum densifolium Brid. & $x$ & CFV, SO, TE & gen & TF & Visnadi \& Vital 5087 \\
\hline *Rosulabryum capillare (Hedw.) J.R. Spence & $x$ & CFV & gen & TF & Visnadi \& Vital 4802 \\
\hline \multicolumn{6}{|l|}{ Calymperaceae } \\
\hline Octoblepharum pulvinatum (Dozy \& Molk.) Mitt. & $x$ & CFV & gen & TF & Visnadi \& Vital 5019 \\
\hline $\begin{array}{l}\text { Syrrhopodon africanus subsp. graminicola (R.S. Williams) } \\
\text { W.D. Reese }\end{array}$ & $x$ & - & gen & TF & $\begin{array}{l}\text { Schäfer-Verwimp \& } \\
\text { Verwimp } 14817\end{array}$ \\
\hline
\end{tabular}


APÊNDICE 1.

(Continua)

\begin{tabular}{|c|c|c|c|c|c|}
\hline Espécies & Fonte & Substrato & $\begin{array}{l}\text { Grupo } \\
\text { ecológico }\end{array}$ & $\begin{array}{l}\text { Forma de } \\
\text { vida }\end{array}$ & Voucher \\
\hline Syrrhopodon gardneri (Hook.) Schwägr. & $x$ & CFV & som & TF & Visnadi \& Vital 5020 \\
\hline Syrrhopodon gaudichaudii Mont. & $x$ & CFV & som & TF & Visnadi \& Vital 4900 \\
\hline Syrrhopodon incompletus var. incompletus & $x$ & CFV & som & TF & Visnadi \& Vital 5036 p.p. \\
\hline Syrrhopodon parasiticus (Sw. Ex Brid.) Besch. & $x$ & $\mathrm{FO}$ & sol & TF & Visnadi \& Vital 5031 p.p \\
\hline $\begin{array}{l}\text { Syrrhopodon prolifer var. cincinnatus (Hampe) W.D. } \\
\text { Reese }\end{array}$ & $x$ & - & gen & TF & Vital \& Buck 20514 \\
\hline Syrrhopodon prolifer var. prolifer & $x$ & $\mathrm{RO}$ & gen & TF & Visnadi \& Vital 4870 \\
\hline Syrrhopodon prolifer var. tenuifolius (Sull.) W.D. Reese & $x$ & SO & gen & TF & Visnadi \& Vital 5163 \\
\hline \multicolumn{6}{|l|}{ Cryphaeaceae } \\
\hline Cryphaea brevipila Mitt. & 7 & - & - & - & - \\
\hline Schoenobryum concavifolium (Griff.) Gangulee & X & - & sol & TF & Vital \& Buck 20556 \\
\hline \multicolumn{6}{|l|}{ Daltoniaceae } \\
\hline Daltonia marginata Griff. & 8 & - & - & - & - \\
\hline *Leskeodon andicola (Spruce ex Mitt.) Broth. & $x$ & SO & som & TP & Visnadi \& Vital 5093 \\
\hline \multicolumn{6}{|l|}{ Dicranaceae } \\
\hline Dicranella hilariana (Mont.) Mitt. & $x$ & $\mathrm{SO}$ & sol & TF & Visnadi \& Vital 4757 \\
\hline *Holomitriopsis laevifolia (Broth.) H. Rob. & X & CFV, SO & som & TF & Visnadi \& Vital 5162 \\
\hline Holomitrium crispulum Mart. & $x$ & CFM & sol & TF & Visnadi \& Vital 4777 \\
\hline Leucoloma serrulatum Brid. & $x$ & CFV, SO & sol & TF & Visnadi \& Vital 5122 \\
\hline \multicolumn{6}{|l|}{ Fissidentaceae } \\
\hline Fissidens asplenioides Hedw. & $x$ & $\mathrm{RO}$ & som & $\mathrm{TF}$ & Visnadi \& Vital 4846 \\
\hline *Fissidens dendrophilus Brugg.-Nann. \& R.A. Pursell & $x$ & SO & som & $\mathrm{F}$ & Visnadi \& Vital 5147 \\
\hline Fissidens rigidulus Hook. f. \& Wilson & $x$ & $\mathrm{RO}$ & som & $\mathrm{F}$ & Visnadi \& Vital 4871 \\
\hline Fissidens scariosus Mitt. & X & $\mathrm{SO}$ & som & $\mathrm{F}$ & Visnadi \& Vital 5146 \\
\hline \multicolumn{6}{|l|}{ Funariaceae } \\
\hline Funaria hygrometrica Hedw. & $x$ & $\mathrm{SO}$ & gen & TF & Visnadi \& Vital 4692 \\
\hline \multicolumn{6}{|l|}{ Hookeriaceae } \\
\hline Crossomitrium patrisiae (Brid.) Müll. Hal. & $x$ & $\mathrm{FO}$ & gen & TP & Visnadi \& Vital 5021 \\
\hline \multicolumn{6}{|l|}{ Hylocomiaceae } \\
\hline *Puiggariopsis aurifolia (Mitt.) M. Menzel & $x$ & CFV & som & $\mathrm{TP}$ & Visnadi \& Vital 4750 \\
\hline \multicolumn{6}{|l|}{ Hypnaceae } \\
\hline Chryso-hypnum diminutivum (Hampe) W.R. Buck & $x$ & CFM, CFV, SO & sol & $\mathrm{TP}$ & Visnadi \& Vital 4818 \\
\hline $\begin{array}{l}\text { *Chryso-hypnum squarrosulum (Cardot) N. Nishim. \& } \\
\text { Ando }\end{array}$ & $x$ & $\mathrm{RO}$ & sol & $\mathrm{TP}$ & Visnadi \& Vital 4948 \\
\hline
\end{tabular}


APÊNDICE 1.

(Continua)

\begin{tabular}{|c|c|c|c|c|c|}
\hline Espécies & Fonte & Substrato & $\begin{array}{l}\text { Grupo } \\
\text { ecológico }\end{array}$ & $\begin{array}{l}\text { Forma de } \\
\text { vida }\end{array}$ & Voucher \\
\hline Mittenothamnium reptans (Hedw.) Cardot & $x$ & CFM, CFV, RO, SO & gen & TP & Visnadi \& Vital 4847 \\
\hline Vesicularia vesicularis (Schwägr.) Broth. & $x$ & CFV, ES, RO, SO & gen & TP & Visnadi \& Vital 5008 \\
\hline \multicolumn{6}{|l|}{ Hypopterygiaceae } \\
\hline Hypopterygium tamariscina (Hedw.) Brid. ex Müll. Hal. & $x$ & CFV, RO & gen & $\mathrm{D}$ & Visnadi \& Vital 4823 \\
\hline Lopidium concinnum (Hook.) Wilson & $x$ & CFV & som & $\mathrm{D}$ & Visnadi \& Vital 4840 \\
\hline \multicolumn{6}{|l|}{ Lembophyllaceae } \\
\hline $\begin{array}{l}\text { Orthostichella pachygastrella (Müll. Hal. ex Angstr.) } \\
\text { B.H. Allen \& Magill }\end{array}$ & $4, \times$ & $\begin{array}{l}\text { CFM, CFV, NP, } \\
\quad \mathrm{RO}, \mathrm{SO}\end{array}$ & gen & $P$ & Visnadi \& Vital 4809 \\
\hline $\begin{array}{l}\text { Orthostichella versicolor (Müll. Hal.) B.H. Allen \& W.R. } \\
\text { Buck }\end{array}$ & $4, X$ & CFV & gen & $P$ & Visnadi \& Vital 5001 \\
\hline Pilotrichella flexilis (Hedw.) Ångström & $x$ & CFV & gen & $\mathrm{P}$ & Visnadi \& Vital 4801 \\
\hline \multicolumn{6}{|l|}{ Leucobryaceae } \\
\hline *Atractylocarpus brasiliensis (Müll. Hal.) R.S. Williams & $x$ & $\mathrm{SO}$ & som & TF & Vital s/n (SP387702) \\
\hline Bryohumbertia filifolia var. filifolia & $x$ & SO & gen & TF & Visnadi \& Vital 5131 \\
\hline Bryohumbertia filifolia var. humilis (Mont.) J.-P. Frahm & $x$ & $\mathrm{SO}$ & gen & TF & Vital s/n (SP387687) \\
\hline Campylopus arctocarpus (Hornsch.) Mitt. & $x$ & CFV, SO & gen & TF & Visnadi \& Vital 5158 \\
\hline Campylopus cryptopodioides Broth. & $x$ & SO, TE & gen & TF & Visnadi \& Vital 4702 \\
\hline Campylopus julicaulis Broth. & $x$ & $\mathrm{sO}$ & gen & TF & Visnadi \& Vital 4698 \\
\hline Campylopus lamellinervis (Müll. Hal.) Mitt. & $x$ & so & gen & TF & Visnadi \& Vital 5176 \\
\hline Campylopus savannarum (Müll. Hal.) Mitt. & $x$ & SO & gen & TF & Visnadi \& Vital 4708 \\
\hline Leucobryum crispum Müll. Hal. & $x$ & SO & gen & TF & Visnadi \& Vital 5108 \\
\hline Leucobryum giganteum Müll. Hal. & $x$ & SO & som & $\mathrm{TF}$ & Visnadi \& Vital 5177 \\
\hline *Leucobryum juniperoideum (Brid.) Müll. Hal. & $x$ & SO & gen & TF & Visnadi \& Vital 5094 \\
\hline \multicolumn{6}{|l|}{ Meteoriaceae } \\
\hline Floribundaria flaccida (Mitt.) Broth. & $4, X$ & CFM, CFV, FO, NP & som & $\mathrm{P}$ & Visnadi \& Vital 4974 \\
\hline Meteorium deppei (Hornsch. ex Müll. Hal.) Mitt. & $4, \times$ & ALV, CFM, CFV & gen & $\mathrm{P}$ & Visnadi \& Vital 4775 \\
\hline Meteorium nigrescens (Hedw.) Dozy \& Molk. & $x$ & $\mathrm{ALV}, \mathrm{CFV}$ & som & $\mathrm{P}$ & Visnadi \& Vital 4970 \\
\hline \multicolumn{6}{|l|}{ Mniaceae } \\
\hline $\begin{array}{l}\text { Pohlia mauiensis (Broth. ex E.B. Bartram) } \\
\text { W. Schultze-Motel }\end{array}$ & $x$ & CFV, ES, RO, SO & som & $\mathrm{TP}$ & Visnadi \& Vital 4831 \\
\hline \multicolumn{6}{|l|}{ Neckeraceae } \\
\hline Homaliodendron piniforme (Brid.) Enroth & X & CFV & som & $\mathrm{D}$ & Vital s/n (SP387880) \\
\hline *Neckera urnigera Müll. Hal. & $x$ & CFV & som & $\mathrm{F}$ & Visnadi \& Vital 4839 \\
\hline Neckeropsis disticha (Hedw.) Kindb. & $x$ & CFV & gen & $\mathrm{F}$ & Visnadi \& Vital 5023 \\
\hline Neckeropsis undulata (Hedw.) Reichardt & $x$ & $\mathrm{CFV}, \mathrm{RO}$ & gen & $\mathrm{F}$ & Visnadi \& Vital 5058 \\
\hline Porotrichum korthalsianum (Dozy \& Molk.) Mitt. & $x$ & CFV, ES, RO & som & $\mathrm{D}$ & Visnadi \& Vital 4903 \\
\hline
\end{tabular}


APÊNDICE 1.

(Continua)

\begin{tabular}{|c|c|c|c|c|c|}
\hline Espécies & Fonte & Substrato & $\begin{array}{l}\text { Grupo } \\
\text { ecológico }\end{array}$ & $\begin{array}{l}\text { Forma de } \\
\text { vida }\end{array}$ & Voucher \\
\hline Porotrichum leucocaulon (Müll. Hal.) Mitt. & $x$ & CFV & som & $\mathrm{D}$ & Visnadi \& Vital 5040 \\
\hline *Porotrichum mutabile Hampe & $x$ & CFV & som & $\mathrm{D}$ & Visnadi \& Vital 5046 \\
\hline Porotrichum substriatum (Hampe) Mitt. & $x$ & CFV, RO & som & $\mathrm{D}$ & Visnadi \& Vital 5003 \\
\hline Thamnobryum fasciculatum (Sw. ex Hedw.) I. Sastre & $x$ & CFV, RO & som & $\mathrm{D}$ & Visnadi \& Vital 5017 \\
\hline \multicolumn{6}{|l|}{ Orthodontiaceae } \\
\hline Hymenodon aeruginosus (Hook. f. \& Wilson) Müll. Hal. & $x$ & CFV & gen & TF & Visnadi \& Vital 5024 \\
\hline \multicolumn{6}{|l|}{ Orthotrichaceae } \\
\hline Groutiella tumidula (Mitt.) Vitt & $x$ & SO & sol & TP & Vital s/n (SP387868) \\
\hline Macrocoma orthotrichoides (Raddi) Wijk \& Margad. & $x$ & CFV, TE & sol & TP & Visnadi \& Vital 5143 \\
\hline Macromitrium cirrosum (Hedw.) Brid. & X & CFV & sol & TP & Visnadi \& Vital 5171 p.p. \\
\hline *Macromitrium longifolium (Hook.) Brid. & $x$ & CFM, CFV & sol & TP & Visnadi \& Vital 4733 \\
\hline Macromitrium punctatum (Hook. \& Grev.) Brid. & $x$ & CFM & sol & $\mathrm{TP}$ & Visnadi \& Vital 4772 \\
\hline Macromitrium richardii Schwägr. & X & CFV & gen & TP & Visnadi \& Vital 4732 \\
\hline Schlotheimia appressifolia Mitt. & $6, \times$ & CFM, CFV & sol & TP & Visnadi \& Vital 4811 \\
\hline Schlotheimia jamesonii (Arn.) Brid. & X & CFM, SO & sol & TP & Visnadi \& Vital 4722 \\
\hline Schlotheimia rugifolia (Hook.) Schwägr. & $x$ & CFV & sol & TP & Visnadi \& Vital 4978 \\
\hline Schlotheimia tecta Hook. f. \& Wilson & $6, x$ & CFV & sol & TP & Vital s/n (SP387884) \\
\hline Schlotheimia torquata (Sw. ex Hedw.) Brid. & $x$ & CFM, CFV & sol & TP & Visnadi \& Vital 4892 \\
\hline \multicolumn{6}{|l|}{ Phyllogoniaceae } \\
\hline Phyllogonium viride Brid. & $x$ & CFM, CFV, SO & som & $P$ & Visnadi \& Vital 5170 \\
\hline \multicolumn{6}{|l|}{ Pilotrichaceae } \\
\hline Callicostella pallida (Hornsch.) Ångström & $x$ & CFM, CFV & som & TP & Visnadi \& Vital 5007 \\
\hline Cyclodictyon albicans (Hedw.) Kuntze & X & CFM, SO & som & TP & Visnadi \& Vital 4793 \\
\hline Cyclodictyon varians (Sull.) Kuntze & $x$ & CFM, SO & som & TP & Visnadi \& Vital 4940 \\
\hline $\begin{array}{l}\text { *Lepidopilidium aureo-purpureum (Geh. \& Hampe) } \\
\text { Broth. }\end{array}$ & $x$ & CFV, FO & som & $\mathrm{TP}$ & Vital s/n (SP387850) \\
\hline Lepidopilum muelleri (Hampe) Hampe & $x$ & CFV, SO & som & TP & Visnadi \& Vital 5014 \\
\hline Lepidopilum scabrisetum (Schwägr.) Steere & $x$ & CFV, SO & som & TP & Visnadi \& Vital 5106 \\
\hline Lepidopilum subsubulatum Geh. \& Hampe & $x$ & CFV & som & TP & Visnadi \& Vital 4962 \\
\hline *Thamniopsis cruegeriana (Müll. Hal.) W.R. Buck & $x$ & - & som & TP & Vital \& Buck 20568 p.p. \\
\hline Thamniopsis incurva (Hornsch.) W.R. Buck & $x$ & CFM, SO & som & TP & Visnadi \& Vital 5092 p.p. \\
\hline Thamniopsis langsdorffii (Hook.) W.R. Buck & $x$ & CFM, SO & gen & TP & Visnadi \& Vital 5107 \\
\hline \multicolumn{6}{|l|}{ Polytrichaceae } \\
\hline Pogonatum pensilvanicum (Bartram ex Hedw.) P. Beauv. & $x$ & SO & sol & TF & Visnadi \& Vital 5084 \\
\hline Polytrichadelphus pseudopolytrichum (Raddi) G.L. Sm. & $x$ & SO & gen & TF & Vital s/n (SP387867) \\
\hline *Polytrichum angustifolium Mitt. & $x$ & $\mathrm{SO}$ & gen & $\mathrm{TF}$ & Visnadi \& Vital 5088 \\
\hline
\end{tabular}


APÊNDICE 1.

(Continua)

\begin{tabular}{|c|c|c|c|c|c|}
\hline Espécies & Fonte & Substrato & $\begin{array}{l}\text { Grupo } \\
\text { ecológico }\end{array}$ & $\begin{array}{l}\text { Forma de } \\
\text { vida }\end{array}$ & Voucher \\
\hline Polytrichum commune Hedw. & $x$ & $\mathrm{SO}$ & gen & TF & Visnadi \& Vital 4705 \\
\hline Pottiaceae & $x$ & $\mathrm{SO}$ & & & Visnadi \& Vital 4756 \\
\hline Barbula indica (Hook.) Spreng. & X & SO & gen & TF & Vital s/n (SP387863) \\
\hline Barbula indica var. indica & 3 & SO & gen & TF & d.l. \\
\hline Chenia leptophylla (Müll. Hal.) R.H. Zander & $x$ & - & gen & TF & Vital \& Buck 20525 \\
\hline Hyophila involuta (Hook.) A. Jaeger & $x$ & ALV, SO & gen & TF & Visnadi \& Vital 4800 \\
\hline Tortella humilis (Hedw.) Jenn. & $x$ & ALV, RO & gen & TF & Visnadi \& Vital 4798 \\
\hline Trichostomum tenuirostre (Hook. \& Taylor) Lindb. & $x$ & $\mathrm{ALV}, \mathrm{RO}, \mathrm{SO}$ & gen & TF & Visnadi \& Vital 5086 \\
\hline \multicolumn{6}{|l|}{ Prionodontaceae } \\
\hline Prionodon densus (Sw. ex Hedw.) Müll. Hal. & $x$ & CFV & som & TF & Visnadi \& Vital 4932 \\
\hline \multicolumn{6}{|l|}{ Pterobryaceae } \\
\hline *Orthostichopsis praetermissa W.R. Buck & $x$ & CFV & gen & $\mathrm{P}$ & Visnadi \& Vital 4950 \\
\hline Orthostichopsis tetragona (Sw. ex Hedw.) Broth. & $x$ & CFM, CFV & som & $\mathrm{P}$ & Visnadi \& Vital 4883 \\
\hline Orthostichopsis tortipilis (Müll. Hal.) Broth. & $x$ & CFM, CFV & som & $P$ & Visnadi \& Vital 4933 \\
\hline Spiridentopsis longissima (Raddi) Broth. & $x$ & $\mathrm{SO}$ & gen & $\mathrm{P}$ & Visnadi \& Vital 4762 \\
\hline \multicolumn{6}{|l|}{ Pylaisiadelphaceae } \\
\hline Isopterygium affusum Mitt. & $8, X$ & - & gen & TP & Vital \& Buck 20436 \\
\hline Isopterygium subbrevisetum (Hampe) Broth. & $8, X$ & CFV, SO & gen & TP & Visnadi \& Vital 5090 \\
\hline Isopterygium tenerifolium Mitt. & $x$ & $\mathrm{SO}$ & gen & TP & Visnadi \& Vital 4879 \\
\hline Isopterygium tenerum (Sw.) Mitt. & $8, x$ & CFM, CFV & gen & TP & Visnadi \& Vital 5009 \\
\hline Wijkia flagellifera (Broth.) H.A. Crum & $x$ & CFV & gen & TP & Visnadi \& Vital 4975 \\
\hline \multicolumn{6}{|l|}{ Racopilaceae } \\
\hline Racopilum intermedium Hampe & $x$ & CFV, RO & som & TP & Visnadi \& Vital 4946 \\
\hline \multicolumn{6}{|l|}{ Rhizogoniaceae } \\
\hline Pyrrhobryum spiniforme (Hedw.) Mitt. & $x$ & CFV, RO, SO & som & TF & Visnadi \& Vital 5091 \\
\hline \multicolumn{6}{|l|}{ Sematophyllaceae } \\
\hline $\begin{array}{l}\text { Acroporium estrellae (Müll. Hal.) W.R. Buck \& A. } \\
\text { Schäfer-Verwimp }\end{array}$ & $x$ & CFV & som & TP & Visnadi \& Vital 4740 \\
\hline $\begin{array}{l}\text { Acroporium exiguum (Broth.) W.R. Buck \& A. } \\
\text { Schäfer-Verwimp }\end{array}$ & $x$ & - & som & TP & Vital \& Buck 20517 \\
\hline Acroporium pungens (Hedw.) Broth. & $x$ & CFV & gen & TP & Vital s/n (SP387841) \\
\hline Aptychopsis pyrrophylla (Müll. Hal.) Wijk \& Margad. & X & - & gen & TP & Vital \& Buck 20481 \\
\hline Paranapiacabaea paulista W.R. Buck \& D.M. Vital & $1, X$ & CFV & gen & $\mathrm{TP}$ & $\begin{array}{l}\text { Schäfer-Verwimp \& } \\
\text { Verwimp } 14781\end{array}$ \\
\hline *Rhaphidostegium cylindrothecium Broth. & $x$ & - & - & TP & Vital \& Buck 20604 \\
\hline Sematophyllum adnatum (Michx.) E. Britton & $x$ & - & gen & TP & Vital \& Buck 20578 \\
\hline
\end{tabular}


APÊNDICE 1.

(Continua)

\begin{tabular}{|c|c|c|c|c|c|}
\hline Espécies & Fonte & Substrato & $\begin{array}{l}\text { Grupo } \\
\text { ecológico }\end{array}$ & $\begin{array}{l}\text { Forma de } \\
\text { vida }\end{array}$ & Voucher \\
\hline Sematophyllum cuspidiferum Mitt. & $x$ & CFV, SO & gen & $\mathrm{TP}$ & Visnadi \& Vital 5123 \\
\hline Sematophyllum galipense (Müll. Hal.) Mitt. & $x$ & - & gen & $\mathrm{TP}$ & Vital \& Buck 20586 \\
\hline $\begin{array}{l}\text { *Sematophyllum leucostomum (Hampe) W.R. } \\
\text { Buck }\end{array}$ & $x$ & - & - & TP & Vital \& Buck 20527 \\
\hline Sematophyllum subpinnatum (Brid.) E. Britton & $x$ & CFV, TE & gen & $\mathrm{TP}$ & Visnadi \& Vital 5142 \\
\hline Sematophyllum subsimplex (Hedw.) Mitt. & $x$ & CFM & gen & $\mathrm{TP}$ & Visnadi \& Vital 5068 \\
\hline \multicolumn{6}{|l|}{ Sphagnaceae } \\
\hline Sphagnum perichaetiale Hampe & $2, x$ & - & gen & TF & Vital \& Buck 20513 \\
\hline \multicolumn{6}{|l|}{ Stereophyllaceae } \\
\hline Eulacophyllum cultelliforme (Sull.) W.R. Buck \& Ireland & $x$ & CFV, RO & sol & TP & Visnadi \& Vital 4904 \\
\hline \multicolumn{6}{|l|}{ Thuidiaceae } \\
\hline *Pelekium minutulum (Hedw.) A. Touw & $x$ & CFM, CFV & gen & TP & Visnadi \& Vital 4942 \\
\hline *Pelekium scabrosulum (Mitt.) A. Touw & $x$ & CFV, SO & gen & $\mathrm{TP}$ & Vital \& Buck 20596 \\
\hline Pelekium schistocalyx (Müll. Hal.) A. Touw & $x$ & $\mathrm{RO}$ & gen & TP & Visnadi \& Vital 4807 \\
\hline Thuidium delicatulum (Hedw.) Schimp. & $x$ & $\mathrm{ES}, \mathrm{RO}, \mathrm{SO}$ & som & $\mathrm{TR}$ & Visnadi \& Vital 4833 \\
\hline Thuidium pseudoprotensum (Müll. Hal.) Mitt. & $x$ & $\mathrm{RO}$ & sol & $\mathrm{TR}$ & Visnadi \& Vital 4995 \\
\hline \multicolumn{6}{|l|}{ MARCHANTIOPHYTA } \\
\hline \multicolumn{6}{|l|}{ Aneuraceae } \\
\hline Riccardia chamedryfolia (With.) Grolle & $x$ & CFV, SO & gen & $\mathrm{TL}$ & Visnadi \& Vital 5207 \\
\hline Riccardia fucoidea (Sw.) Schiffn. & $x$ & CFM, SO & gen & $\mathrm{TL}$ & Vital s/n (SP387890) \\
\hline Riccardia metzgeriiformis (Steph.) R.M. Schust. & $x$ & CFM, SO & gen & $\mathrm{TL}$ & Visnadi \& Vital 4700 \\
\hline \multicolumn{6}{|l|}{ Balantiopsidaceae } \\
\hline Isotachis aubertii (Schwägr.) Mitt. & $x$ & SO & gen & $\mathrm{TP}$ & Visnadi \& Vital 4693 \\
\hline *Isotachis multiceps (Lindenb. \& Gottsche) Gottsche & $x$ & SO & gen & $\mathrm{TP}$ & Visnadi \& Vital 4712 \\
\hline Neesioscyphus homophyllus (Nees) Grolle & $x$ & SO & gen & TP & Visnadi \& Vital 5159 \\
\hline \multicolumn{6}{|l|}{ Calypogeiaceae } \\
\hline Calypogeia peruviana Nees \& Mont. & $x$ & CFV, SO & som & $\mathrm{TP}$ & Visnadi \& Vital 5144 \\
\hline \multicolumn{6}{|l|}{ Cephaloziellaceae } \\
\hline *Cephaloziella divaricata (Sm.) Schiffn. & $x$ & SO & gen & $\mathrm{TP}$ & Vital s/n (SP387686) \\
\hline \multicolumn{6}{|l|}{ Frullaniaceae } \\
\hline Frullania arecae (Spreng.) Gottsche & $x$ & $\mathrm{CFV}, \mathrm{RO}$ & sol & $\mathrm{TP}$ & Visnadi \& Vital 4803 p.p. \\
\hline Frullania brasiliensis Raddi & $x$ & CFV, CFM, SO & sol & $\mathrm{TP}$ & Visnadi \& Vital 5130 \\
\hline Frullania caulisequa (Nees) Nees & $x$ & CFV & sol & $\mathrm{TP}$ & Visnadi \& Vital 5206 \\
\hline Frullania setigera Steph. & $x$ & CFV, NP & sol & TP & Visnadi \& Vital 4735 \\
\hline \multicolumn{6}{|l|}{ Geocalycaceae } \\
\hline Saccogynidium caldense (Ångstr.) Grolle & $x$ & SO & gen & $\mathrm{TP}$ & Visnadi \& Vital 5129 \\
\hline
\end{tabular}


APÊNDICE 1.

(Continua)

\begin{tabular}{|c|c|c|c|c|c|}
\hline Espécies & Fonte & Substrato & $\begin{array}{l}\text { Grupo } \\
\text { ecológico }\end{array}$ & $\begin{array}{l}\text { Forma de } \\
\text { vida }\end{array}$ & Voucher \\
\hline \multicolumn{6}{|l|}{ Herbertaceae } \\
\hline $\begin{array}{l}\text { Herbertus juniperoideus ssp. bivittatus (Spruce) Feldberg } \\
\text { \& Heinrichs }\end{array}$ & $x$ & CFV, SO & som & TF & Visnadi \& Vital 5161 \\
\hline \multicolumn{6}{|l|}{ Jamesoniellaceae } \\
\hline Syzygiella rubricaulis (Nees) Steph. & $x$ & CFV & gen & C & Vital s/n (SP387911) \\
\hline \multicolumn{6}{|l|}{ Lejeuneaceae } \\
\hline $\begin{array}{l}\text { Anoplolejeunea conferta (C. F. W. Meissn. ex Spreng.) } \\
\text { A. Evans }\end{array}$ & $x$ & CFM, CFV & gen & TP & Visnadi \& Vital 5188 \\
\hline Archilejeunea parviflora (Nees) Schiffn. & $x$ & CFV & som & TP & Visnadi \& Vital 4863 \\
\hline $\begin{array}{l}\text { *Brachiolejeunea leiboldiana (Gottsche \& Lindenb.) } \\
\text { Schiffn. }\end{array}$ & $x$ & CFV & gen & TP & Visnadi \& Vital 5190 \\
\hline Bryopteris diffusa (Sw.) Nees & $x$ & CFV & som & $\mathrm{D}$ & Visnadi \& Vital 5033 \\
\hline Bryopteris filicina (Sw.) Nees & $x$ & CFV, RO & som & $\mathrm{D}$ & Visnadi \& Vital 5119 \\
\hline Ceratolejeunea cornuta (Lindenb.) Schiffn. & $x$ & CFV & gen & TP & Visnadi \& Vital 4731 \\
\hline Ceratolejeunea laetefusca (Austin) R.M. Schust. & $x$ & CFV & gen & $\mathrm{TP}$ & Visnadi \& Vital 5175 p,p. \\
\hline Cheilolejeunea acutangula (Nees) Grolle & $x$ & CFV & gen & TP & Visnadi \& Vital 5125 \\
\hline Cheilolejeunea unciloba (Lindenb.) Malombe & $x$ & CFV & gen & TP & Visnadi \& Vital 5189 p.p. \\
\hline $\begin{array}{l}\text { Cheilolejeunea xanthocarpa (Lehm. \& Lindenb.) } \\
\text { Malombe }\end{array}$ & $x$ & CFV & sol & TP & Visnadi \& Vital 5195 \\
\hline Cololejeunea cardiocarpa (Mont.) A. Evans & $x$ & $\mathrm{FO}$ & gen & TP & Visnadi \& Vital 5186 p.p. \\
\hline Cololejeunea obliqua (Nees \& Mont.) Schiffn. & $x$ & $\mathrm{FO}$ & gen & TP & Visnadi \& Vital 5022 \\
\hline Cololejeunea vitalana Tixier & $5, x$ & - & - & $\mathrm{TP}$ & $\begin{array}{l}\text { Schäfer-Verwimp \& } \\
\text { Verwimp } 12660\end{array}$ \\
\hline Diplasiolejeunea cavifolia Steph. & $3, x$ & $\mathrm{FO}$ & sol & TP & Visnadi \& Vital 4909 p.p. \\
\hline Diplasiolejeunea rudolphiana Steph. & $x$ & $\mathrm{FO}$ & sol & $\mathrm{TP}$ & Visnadi \& Vital 4684 \\
\hline Drepanolejeunea bidens (Steph.) A. Evans & $x$ & $\mathrm{FO}$ & gen & TP & Visnadi \& Vital 5185 \\
\hline Drepanolejeunea biocellata A. Evans & $x$ & $\mathrm{FO}$ & gen & TP & Visnadi \& Vital 5101 \\
\hline Drepanolejeunea mosenii (Steph.) Bischl. & $x$ & CFV, FO & gen & TP & Visnadi \& Vital 5183 \\
\hline *Frullanoides densifolia Raddi & $x$ & CFV & sol & TP & Vital s/n (SP387685) \\
\hline Harpalejeunea subacuta A. Evans & $x$ & CFV & gen & TP & Visnadi \& Vital 5205 \\
\hline Lejeunea controversa Gottsche & $x$ & CFV & gen & TP & Visnadi \& Vital 5006 \\
\hline Lejeunea cristulata (Steph.) E. Reiner \& Goda & $x$ & CFV & som & TP & Visnadi \& Vital 5184 \\
\hline Lejeunea flava (Sw.) Nees & $x$ & CFV, RO, SO & gen & TP & Visnadi \& Vital 5182 \\
\hline Lejeunea glaucescens Gottsche & $x$ & $\mathrm{FO}$ & som & TP & Visnadi \& Vital 4909 p.p. \\
\hline Lejeunea grossitexta (Steph.) E. Reiner \& Goda & $x$ & CFV & gen & TP & Visnadi \& Vital 5203 \\
\hline *Lejeunea minutiloba A. Evans & $x$ & CFV & som & TP & Visnadi \& Vital 5200 \\
\hline
\end{tabular}


APÊNDICE 1.

(Continua)

\begin{tabular}{|c|c|c|c|c|c|}
\hline Espécies & Fonte & Substrato & $\begin{array}{l}\text { Grupo } \\
\text { ecológico }\end{array}$ & $\begin{array}{l}\text { Forma de } \\
\text { vida }\end{array}$ & Voucher \\
\hline *Lejeunea raddiana Lindenb. & $x$ & $\mathrm{FO}$ & som & TP & Visnadi \& Vital 5102 \\
\hline Lopholejeunea subfusca (Nees) Schiffn. & $x$ & CFV & sol & $\mathrm{TP}$ & Visnadi \& Vital 4862 \\
\hline Marchesinia brachiata (Sw.) Schiffn. & $x$ & $\mathrm{RO}$ & sol & $\mathrm{TR}$ & Visnadi \& Vital 4803 p.p. \\
\hline *Mastigolejeunea auriculata (Wilson) Schiffn. & $x$ & CFM & gen & TP & Vital s/n (SP387701) \\
\hline Microlejeunea bullata (Taylor) Steph. & $x$ & $\mathrm{FO}$ & gen & TR & Visnadi \& Vital 4688 \\
\hline Odontolejeunea lunulata (Weber) Schiffn. & $x$ & $\mathrm{FO}$ & gen & $\mathrm{TP}$ & Visnadi \& Vital 5100 \\
\hline Omphalanthus filiformis (Sw.) Nees & $x$ & CFM, CFV, RO, SO & gen & $\mathrm{TP}$ & Visnadi \& Vital 4806 \\
\hline Schiffneriolejeunea polycarpa (Nees) Gradst. & $x$ & CFV & sol & $\mathrm{TR}$ & Visnadi \& Vital 5180 \\
\hline Taxilejeunea isocalycina (Nees) Steph. & $x$ & CFV & som & $\mathrm{TP}$ & Visnadi \& Vital 5070 \\
\hline Taxilejeunea lusoria (Lindenb. \& Gottsche) Steph. & $x$ & CFV & som & $\mathrm{TP}$ & Visnadi \& Vital 5202 \\
\hline \multicolumn{6}{|l|}{ Lepidoziaceae } \\
\hline Bazzania heterostipa (Steph.) Fulford & $x$ & CFV & gen & $\mathrm{TR}$ & Visnadi \& Vital 5192 \\
\hline Bazzania hookeri (Lindenb.) Trevis. & $x$ & CFV, SO & gen & TR & Visnadi \& Vital 5155 \\
\hline Bazzania longistipula (Lindenb.) Trevis. & $x$ & SO & gen & $\mathrm{TR}$ & Visnadi \& Vital 5160 \\
\hline Kurzia capillaris (Sw.) Grolle & X & SO & som & TP & Visnadi \& Vital 5116 \\
\hline $\begin{array}{l}\text { Lepidozia inaequalis (Lehm. \& Lindenb.) Lehm. \& } \\
\text { Lindenb. }\end{array}$ & $x$ & CFV & gen & $\mathrm{TR}$ & Visnadi \& Vital 4765 \\
\hline Paracromastigum pachyrhizum (Nees) Fulford & $x$ & SO & gen & $\mathrm{TR}$ & Visnadi \& Vital 5112 p.p. \\
\hline Telaranea nematodes (Gottsche ex Austin) M.A. Howe & $x$ & CFM, SO & som & $\mathrm{TP}$ & Visnadi \& Vital 5114 \\
\hline \multicolumn{6}{|l|}{ Lophocoleaceae } \\
\hline Chiloscyphus martianus (Nees) J.J. Engel \& R.M. Schust. & $x$ & CFV, CFM, SO & gen & TP & Visnadi \& Vital 5010 \\
\hline \multicolumn{6}{|l|}{ Metzgeriaceae } \\
\hline Metzgeria albinea Spruce & $x$ & CFV, CFM, RO, TE & gen & $\mathrm{TL}$ & Visnadi \& Vital 5124 \\
\hline Metzgeria aurantiaca Steph. & $x$ & $\mathrm{FO}$ & gen & $\mathrm{TL}$ & Visnadi \& Vital 5081 \\
\hline Metzgeria consanguinea Schiffn. & $x$ & CFV & gen & $\mathrm{TL}$ & Visnadi \& Vital 5078 \\
\hline Metzgeria myriopoda Lindb. & $x$ & CFV & gen & $\mathrm{TL}$ & Vital s/n (SP387694) \\
\hline \multicolumn{6}{|l|}{ Pallaviciniaceae } \\
\hline Pallavicinia lyellii (Hook.) S. F. Gray & $x$ & $\mathrm{RO}$ & som & $\mathrm{TL}$ & Visnadi \& Vital 4872 \\
\hline Symphyogyna brasiliensis (Nees) Nees \& Mont. & $x$ & SO & som & $\mathrm{TL}$ & Visnadi \& Vital 5113 \\
\hline \multicolumn{6}{|l|}{ Plagiochilaceae } \\
\hline *Plagiochila adiantoides (Sw.) Lindenb. & X & CFV & som & $\mathrm{F}$ & Vital s/n (SP387910) \\
\hline Plagiochila bifaria (Sw.) Lindenb. & X & CFV & som & $\mathrm{F}$ & Visnadi \& Vital 4891 \\
\hline Plagiochila bunburii Taylor & $x$ & CFV, RO & som & $\mathrm{F}$ & Visnadi \& Vital 4919 \\
\hline Plagiochila corrugata (Nees) Nees \& Mont. & $x$ & CFV, TE & som & $\mathrm{F}$ & Visnadi \& Vital 4955 \\
\hline Plagiochila disticha (Lehm. \& Lindenb.) Lindenb. & $x$ & CFV & som & $\mathrm{F}$ & Visnadi \& Vital 5127 \\
\hline Plagiochila gymnocalycina (Lehm. \& Lindenb.) Lindenb. & $x$ & $\mathrm{CFV}, \mathrm{FO}, \mathrm{RO}, \mathrm{SO}$ & som & $\mathrm{F}$ & Visnadi \& Vital 5005 \\
\hline
\end{tabular}


APÊNDICE 1.

(Conclusão)

\begin{tabular}{l|c|c|c|c|c}
\hline \multicolumn{1}{c|}{ Espécies } & Fonte & Substrato & $\begin{array}{c}\text { Grupo } \\
\text { ecológico }\end{array}$ & $\begin{array}{c}\text { Forma de } \\
\text { vida }\end{array}$ & Voucher \\
\hline Plagiochila martiana (Nees) Lindenb. & $\times$ & CFV, FO, RO & som & F & Visnadi \& Vital 5050 \\
\hline Plagiochila patentissima Lindenb. & $\times$ & CFV, RO, SO & som & F & Visnadi \& Vital 5077 \\
\hline Plagiochila patula (Sw.) Lindenb. & $\times$ & CFV & som & F & Visnadi \& Vital 4914 \\
\hline Plagiochila subplana Lindenb. & $\times$ & CFV, ES, RO & som & F & Visnadi \& Vital 4906 \\
\hline Porellaceae & $\times$ & CFV, RO & som & TP & Visnadi \& Vital 4850 \\
\hline Porella brasiliensis (Raddi) Schiffn. & $\times$ & CFV, RO & gen & TP & Visnadi \& Vital 5049 \\
\hline Porella swartziana (Weber) Trevis. & & & & & \\
\hline Radulaceae & $\times$ & CFV & som & TP & Visnadi \& Vital 5025 \\
\hline Radula gottscheana Tayl. & $\times$ & CFV, RO, SO & gen & TP & Visnadi \& Vital 5199 \\
\hline Radula recubans Tayl. & $\times$ & CFV & gen & TP & Vital s/n (SP387695) \\
\hline *Radula sinuata Gottsche ex Steph. & $\times$ & CFV & gen & TP & Vital s/n (SP387696) \\
\hline Radula tectiloba Steph. & $\times$ & CFV & som & TP & Vital s/n (SP387907 \\
\hline *Radula tenera Mitt. ex Steph. & $\times$ & CFV, RO & gen & TP & Visnadi \& Vital 4890 \\
\hline *Radula voluta Tayl. ex Gottsche et al. & & & & & \\
\hline Scapaniaceae & $\times$ & - & gen & TP & Vital \& Buck 20519 p.p. \\
\hline *Scapania portoricensis Hampe \& Gottsche & & RO & som & TP & Vital \& Buck 20502 \\
\hline Trichocoleaceae & & & & & \\
\hline Trichocolea flaccida (Spruce) J.B. Jack \& Steph. & $\times$ & & & \\
\hline
\end{tabular}


